\title{
sciendo
}

\section{The Structure of Selected Basic Acrobatic Jumps}

\author{
by \\ Henryk Król' ${ }^{1}$, Małgorzata Klyszcz-Morciniec², Bogdan Bacik ${ }^{1}$
}

The aim of this study was to investigate the relationships between the internal and external structure of basic acrobatic jumps. Eleven healthy elite artistic gymnasts (9 female, 2 male) participated in this study. Participants performed the following basic 'acrobatic' jumps: a tucked backward somersault (TS), a piked backward somersault (PS), and a countermovement jump (CMJ). Furthermore, female gymnasts also performed the backward handspring (HS), taking off and then landing on their hands in the same place - a specific jump only for women. All jumps were initiated from a stationary upright posture and with an arms swing. Six infrared cameras, synchronized with a module for wireless measurement of the electrical activity of eight muscles, and the force plate were used. Infrared camera-recordings were made in order to obtain kinematic variables describing the movement structure of the acrobatic jumps. These variables may explain the characteristics of muscle activation (the internal structure of the movement) and ground reaction force (the external-kinetic structure of the movement). However, for various technical reasons, it was not possible to register all the specified jumps in the protocol. Moreover, the distribution normalities, estimated by the Kolmogorov-Smirnov test, differed between variables. Therefore, to compare the data, the pair-wise nonparametric Wilcoxon Signed-Ranks Test was applied. The CMJ showed the highest level of vertical impulse, velocity, and displacement followed by the TS, PS, and HS. In the take-off phase of acrobatic jumps with rotation the average muscle activation levels of the biceps femoris were significantly higher and of the rectus femoris significantly lower than in the countermovement jump.

Key words: Smart-E measuring system, gymnastics, movement structure, movement analysis.

\section{Introduction}

Biomechanical research in gymnastics has often been haphazard. Very little of the research reveals attempts to develop biomechanical "critical features" of the movement (Bartlett, 2009) for the analyzed skills. The purpose of most research appears rather to have concentrated on what is considered contemporary good performances, and occasionally, to compare those with worse performances. However, identification of these critical features is probably the most important task that a qualitative or quantitative analyst needs to face.

One of the primary tasks of sports biomechanics is the quantitative analysis of movement techniques to facilitate the learning of motor skills. Quantitative biomechanical movement analysis primarily uses temporal characteristics of mechanical variables (Droszez et al., 2016). However, to effectively communicate with athletes, coaches often refer to the so-called 'movement features' (Schnabel, 1998). These features are primarily used in a qualitative analysis of sports activities. Movement features can also be useful in sports technique studies, because they have certain defined measures. In this paper, as a basis for consideration and analysis, an eightelement, hierarchical and modified classification of movement features recommended by Schnabel (1998) was employed. Three groups of movement features can be distinguished here: the parent feature (the general basic structure), complex features (the rhythm, and the motion coupling), and the elementary features (fluency i.e., fluidity, accuracy i.e., exactitude, constancy i.e., repeatability, the rate of the movement, amplitude i.e., extensiveness of motion). The most informative and useful feature in the evaluation of

1 - Institute of Sport Sciences, The Jerzy Kukuczka Academy of Physical Education, Katowice, Poland.

2 - Former student, The Jerzy Kukuczka Academy of Physical Education, Katowice, Poland. 
the movement, is the structure of the movement (Schnabel, 1998). To the observer, only the spatiotemporal structure of the movement is available. Assessment of this parent feature and "generalized category", which is based only on visual observation, is relatively simple, but contains little information (Schnabel, 1998). Therefore, research on sport techniques should cover both the causes of motion (the internal and external-kinetic structure of the movement) as well as the external-kinematic structure that shows the effects of motion, i.e. all temporal characteristics of the movement (Krol and Golas, 2017).

Biomechanics is the basic sports science focusing on movement techniques. This is especially important in gymnastics where performance techniques of the best gymnasts become models for others to follow. A coach's ability to direct technical and physical training for these specific skills is enhanced when thorough descriptions of the skills are available ('movement features', in particular 'critical features').

In scientific research on movement techniques of motor skills and in recording and/or the measurement of different features and variables of movement, various accessible biomechanical methods are utilized. In previous studies, we applied a set of methodological research tools to study the structure of movement in the flat bench press (Golas and Krol, 2014; Krol et al., 2010), and acrobatic jumps (Krol et al., 2014, 2016; Krol and Klyszcz-Morciniec, 2017). An understanding of both the internal (muscle activation) and external (kinematic and kinetic) structure of these sport activities was acquired by simultaneous application of several devices, such as: a force plate, electromyography, and cameras. In this study, our goals were the same for the following basic standing 'acrobatic' jumps: a tucked backward somersault (TS), a piked backward somersault (PS), a backward handspring (HS), and a countermovement jump (CMJ).

The structure of these acyclic movements generally consists of the initial, main and final phases. However, for the jumps with rotation (TS, PS, and HS), the names of the phases are specific: countermovement, take-off, flight and landing phases. The countermovement is a specific type of the initial phase aimed to create optimal conditions for the implementation of the main phase. This is achieved by the pre-stretching of muscles of both legs (e.g. triceps, quadriceps, gluteus maximus) and the trunk (e.g. erector spinae pars lumborum). The pre-stretching of the muscles increases its elastic energy, which is called, the stretchshortening cycle - SSC (McNeal et al., 2007). The SSC, according to Bartlett (2009), is a common sequence of joint actions in which an eccentric (lengthening) muscle contraction, or pre-stretch, precedes a concentric (shortening) muscle contraction. Both the take-off and the flight are the main phases since the main task is performed then. The main task involves body rotation about the free transverse axis. The purpose of the take-off is to provide the projection velocity needed to lift the body and the angular momentum required to perform a rotary motion. The flight includes the tucking action (during the ascent of the body) and the opening out action (during the descent). The effectiveness of the flight phase is determined by the skillful use of the principle of the conservation of angular momentum. The first aim of the landing is to overcome both the momentum and the angular momentum of the body. The second aim is to protect the joints of the lower limbs from injury and to restore the standing position.

Both male and female artistic gymnasts must be able to properly perform basic motor skills such as backward somersaults and backward handsprings, as well as countermovement jumps. Proper performance of these gymnastic skills requires an appropriate level of fitness and coordination abilities. These skills are usually performed as parts of acrobatic tumbling sequences, in which the take-off plays an essential role. Most of the complicated tumbling combinations are performed backwards due to the advantageous anatomical conditions for the backward take-off (Knoll, 1996). Backward takeoffs are one of the most important and frequently used components of a floor exercise routine in artistic gymnastics. The backward take-off initiates the linear and rotational impulses essential for the optimization of take-off velocities by attaining a large amount of kinetic energy necessary to achieve a suitable magnitude of momentum and angular momentum (Geiblinger et al., 1995). In the countermovement jump, the kinetic energy is necessary only to achieve a large magnitude of linear momentum. The focus of our analysis included sports technical solutions for energy production during the take-off phase for the 
following standing 'acrobatic' jumps: a TS, a PS, a HS, and a CMJ.

At the end of the countermovement phase there is a decrease in the angle between segments of lower extremities. The leg muscles (triceps, quadriceps, gluteus maximus) are stretched and part of the kinetic energy is transformed into potential energy of elasticity of leg muscles. This form of mechanical energy is then, by contraction of leg muscles, transformed back into kinetic energy which is at its maximum during the takeoff phase (SSC).

As with all ballistic movements, a ballistic movement is initiated by muscle activity in one muscle group, continues during a 'coasting' period with no muscle activation, and terminates by deceleration caused by the antagonist muscle group or by passive tissue structures, such as ligaments (Bartlett, 2009). In gymnastics, the mechanics for initiating acrobatic jumps follows a specific movement pattern. According to George (2014) prior to the jump, a vigorous upwardthrowing action of the arms occurs which sets the stage for the kinetic chain sequencing of the legs. Since it is more difficult to start a movement of a body than to keep a body in motion, a powerful extension at the hips provides the fundamental force. This initiation of hip-joint extension is the cue to begin immediate, forceful knee-joint extension, which in turn is the cue to plantarflexion of the ankle joints. The specific hip-kneeankle sequencing order must occur to ensure the most effective momentum transfer (Bobbert and van Ingen Schenau, 1988; George, 2014; Pandy et al., 1990). In our study this initiation of hip-joint extension has also become the basis for the separation of the countermovement phase from the take-off phase. The end of the take-off phase occurs when the vertical component of ground reaction force is zero, while the end of the flight phase follows at the instant of touch-down.

The most comprehensive studies on backward take-offs on the floor have been conducted by Knoll and Krug (1989), Hwang et al. (1990), Bruggemann (1994), Geiblinger et al.(1995), and Hedbavny and Kalichova (2011). Unfortunately, there is still a lack of professional research analyzing the full gymnastic movement structures in detail, i.e., both the internal and external (kinetic and kinematic) structure of the movement. Taking into consideration the existing knowledge and its deficiencies, the first aim of this study was to investigate the internal and external structure of basic acrobatic jumps. The second aim was to investigate the relationships that characterize these structures, according to the type of the acrobatic jump (with or without rotation). Based on previous studies (Krol et al., 2014; Krol and Klyszcz-Morciniec, 2017), we hypothesized that: 1) differences in the electro-activity pattern of the same muscles during three defined phases exist between acrobatic jumps with (TS, PS, HS) and without rotation (CMJ), 2) in the take-off phase of the TS, PS and HS the average muscle activity level of the biceps femoris is significantly higher and of the rectus femoris significantly lower compared to the CMJ. Understanding these characteristics could facilitate appropriate technical and physical training for gymnasts performing these simple standing acrobatic jumps.

\section{Methods}

\section{Participants}

Eleven healthy artistic gymnasts (9 female, 2 male), all members of the Polish national gymnastics team participated in the study. Participants comprised a sample of highly competitive national level gymnasts who demonstrated proficiency in performing the analyzed skills. The physical characteristics of participants are presented in Table 1.

Gymnasts were informed about the nature of the study and prior to data collection they were required to sign a consent form according to human subject regulations. Parent or guardian consent was required for those younger than 18 . The research project was approved by the Ethics Committee for Scientific Research of the Jerzy Kukuczka Academy of Physical Education in Katowice, Poland. Gymnasts were free of injury and testing was performed during the competitive period.

\section{Instrumentation and data collection}

Acrobatic jumps.

All participants were tested under the same conditions, in a laboratory setting. Before starting the evaluations, gymnasts were asked to warm up with their own routine for 'typical' training. After a general warm-up, participants performed their own 10-min stretching program. Before performing the jumps, each participant was given detailed instructions and allowed a brief period of practice. Each participant was recorded while performing the following basic standing 'acrobatic' jumps: a tucked backward 
somersault (TS), a piked backward somersault (PS), and a countermovement jump (CMJ). Furthermore, female gymnasts also performed a backward handspring (HS), taking off and then landing on their hands in place, which is a specific jump for women only. All jumps were initiated from a stationary upright posture and with an arm swing. The order of the jumps was randomized and 2-3 min rest intervals were allowed between subsequent trials.

Smart measuring system. A multi-dimensional movement analysis was made with the measuring system Smart-E (BTS Bioengineering, Italy). The system consisted of six infrared cameras with a frequency of $120 \mathrm{~Hz}$, synchronized with a module for wireless measurement of the electrical activity of the muscle called Pocket EMG (BTS Bioengineering, Italy), and the force plate (Kistler Instruments Corp., Switzerland). Data from the force plate, the module for electromyographic measurement, and the cameras were collected simultaneously, and transmitted immediately to the computer via Wi-Fi network.

Three and two dimensional kinematics.

Infrared camera-recordings were made in order to obtain kinematic variables describing the movement structure of the acrobatic jumps. These variables may explain the characteristics of muscle activation. Exact spatial accuracy was achieved by attaching the test retro reflective (passive) markers (diameter of $19 \mathrm{~mm}$ ) to the body of the participant. Twenty-two passive markers were placed on various parts of both sides of the body thus making it possible to determine the centre of mass (COM) of the whole body. These were: tuber calcanei, os metatarsale $V$, malleolus lateralis, epicondylus lateralis femoris, trochanter major, $3 \mathrm{~cm}$ above ala ossis ilii, acromion, epicondylus lateralis humeri, between the processus styloideus radii and processus styloideus ulnae, between the second and third caput ossis metacarpale, and $1 \mathrm{~cm}$ before the meatus acusticus externus. 3D modeling as well as calculations of variables were performed with Smart software (Smart Tracker, Smart Capture, and Smart Analyzer; BTS Bioengineering, Italy). The technical accuracy of the system's measurements after the calibration process was $0.4 \mathrm{~mm}$ i.e. the distance between two markers in 3D. However, in quantitative analysis of 'acrobatic' jumps (TS, PS, HS and CMJ) we only used data which formed a sagittal plane. The inertial variables (masses and lengths of individual body parts) were estimated with the inertia coefficients proposed by Zatsiorsky et al. (1981). The moment of inertia $(I)$, angular velocity $(\omega)$, and angular momentum $(H)$ around the centre of mass in the sagittal plane was calculated according to Lukin (1964, 1966). The vertical velocity $\left(v_{y}\right)$ of the COM was calculated at the moment of the take-off. Vertical displacement $\left(d_{y}\right)$ of this point during the flight phase was also studied. The take-off angle (the angle of the weight centre velocity measured in relation to horizontal at the instant the feet leave the ground $-\lambda)$, ankle angle $(\alpha)$, knee angle $(\beta)$, hip angle $(\gamma)$ and shoulder joint angle $(\delta)$ were also analyzed and compared between jumps. Furthermore, the countermovement time $\left(t_{\mathrm{cm}}\right)$, the take-off time $\left(t_{\text {take-off }}\right)$, and the flight phase time ( $\left.t_{\text {flight }}\right)$ were analyzed.

Electromyography.

Multi-channel electromyography (EMG) can serve in studies of muscular coordination, thus enabling specific evaluation of jumping skills. Muscle activity was assessed using the BTS Pocket EMG (BTS Bioengineering, Italy). The electromyography signals were monitored using H124SG disposable electrodes. Two surface electrodes were placed $2 \mathrm{~cm}$ apart over the motor activation points of the anterior tibialis (AT), medial gastrocnemius (MG), rectus femoris (RF), biceps femoris $(\mathrm{BF})$, rectus abdominis (RA), gluteus maximus $(\mathrm{GM})$, erector spinae (ES), and anterior deltoideus $(\mathrm{AD})$, in accordance with European Recommendations for Surface Electromyography - SENIAM, and secured with athletic tape. All electrodes were placed on the right side of the gymnast's body. The surface electrodes were used to obtain the muscle activation characteristics of the gymnast during the countermovement, take-off, and flight (airborne) phases of each jump. Before electrode placement, the skin surface was vigorously scrubbed with an alcohol swab. All electrodes remained in place until the end of all trials. Cables from the electrodes to the transmitter were secured to the gymnast with athletic tape to minimize distraction to the gymnast and interference to the EMG signal. The transmitter was placed in a belt pack worn snugly around the gymnast's waist. EMG signals were taken at a $1 \mathrm{kHz}$ sample rate. All active channels had the same measuring range and were fitted to the gymnast (typically $+/-5 \mathrm{mV}$ ). Analog signals were converted to digital with 16 bit sampling resolution and collected on the measuring unit.

Electromyography data reduction and testing procedures. The raw EMG signal was filtered (pass-band Butterworth filter, 10-250 Hz). Next, the full-wave was rectified and smoothed using the root-mean-square (RMS) method with a $100 \mathrm{~ms}$ mobile window. The average measurement was calculated from the RMS 
EMG in millivolt, for the countermovement, take-off and flight phases of each acrobatic jump. For this purpose, we used the previously mentioned Smart Analyzer software (BTS Bioengineering, Italy). After the measurement session, to compare muscle activity of participants and give biologically meaningful data, maximal normalization contractions were performed for each muscle, the so-called Maximum Voluntary Contraction (MVC) normalization. This required the participant to contract each muscle against manual resistance provided by the experimenter for the maximum of $3 \mathrm{~s}$. MVC positions for the individual muscles were chosen based on Konrad's (2006) proposals. The highest activity levels in EMG during a $100 \mathrm{~ms}$ interval achieved in the MVC test, reflected the peak EMG of the muscles under isometric conditions. The maximal peak muscle activity was calculated and recorded from a suitable maximum contraction and all subsequent muscle activity was expressed as a percentage of this MVC peak. MVC data were processed in the same way as myoelectric data from the jumps.

Force plate.

Gymnasts were instructed to perform all jumps from a standing position with a take-off from and a landing on a $60 \times 40 \mathrm{~cm}$ Kistler force plate (Kistler Instruments, Switzerland. Type 9281C). Sampling frequency was $240 \mathrm{~Hz}$, and the measuring range was set between 10 to $20 \mathrm{kN}$. A vertical and horizontal (anterior-posterior) component of the ground reaction force (GRF) was recorded. To calculate the vertical and horizontal force impulse $\left(F \cdot t_{y}, F \cdot t_{x}\right)$, the COM velocity $\left(v_{y}, v_{x}\right)$, and displacement $\left(d_{y}, d_{x}\right)$, computer software was implemented (MATLAB).

\section{Statistical Analysis}

Initially the basic methods of descriptive statistics were applied to data obtained from particular measurements. All data are reported as mean \pm standard deviation (SD). The distributions normalities, estimated by the KolmogorovSmirnov test, differed between variables. Furthermore, for technical reasons only 5 female gymnasts performed all the specified jumps in the protocol, and in view of feminine specificity of the back handspring, i.e., landing on hands in the same place as the take-off, the male gymnasts did not perform this jump. Therefore, the nonparametric Wilcoxon Signed-Ranks test was applied to compare the data pair-wise. The level of significance was set at $p \leq 0.05$. All statistical analyses were performed using Statistica v. 7 package (StatSoft, Inc.).

\section{Results}

The execution assessment of selected acrobatic skills was performed by comparing the tucked somersault (TS) with three other acrobatic jumps (CMJ, PS and HS).

Bioelectrical activity of muscles - internal structure of standing acrobatic jumps. Temporarily normalized RMS EMG [\% of MVC] linear envelopes during four acrobatic jumps for eight muscle are plotted in Figures 1-3.

Tucked backward somersault vs. countermovement jump. A comparison of EMG patterns in the set of muscles between the tucked backward somersault and the countermovement jump performed by the same group of gymnasts indicates large differences (Figures1A and 1B; Table 2). Statistically significant differences $(p<$ 0.05 ) in average muscle activation levels of five muscles were especially evident in the countermovement and flight phases. In four cases these were the same muscles. The mean muscle activation levels were somewhat higher for the TS than the CMJ (during the countermovement phase it equaled for MG $69.8 \pm 54.4 \%$ MVC and 30.9 $\pm 25.5 \%$ MVC, respectively; for RF $7.2 \pm 8.7 \%$ MVC and $3.4 \pm 2.7 \% \mathrm{MVC}$, respectively; for BF $13.3 \pm 7.7 \%$ MVC and 7.3 $\pm 4.8 \%$ MVC, respectively; for RA 10.4 $\pm 14.3 \% \mathrm{MVC}$ and $3.3 \pm 2.5 \% \mathrm{MVC}$, respectively; and in the flight phase for AT $76.1 \pm 17.5 \%$ MVC and $35.2 \pm 24.7 \%$ MVC, respectively; for RF $56.9 \pm 25.4 \%$ MVC and $25.2 \pm 24.2 \%$ MVC, respectively; for BF $40.0 \pm 12.8 \% \quad$ MVC and $15.7 \pm 8.9 \%$ MVC, respectively; for RA $76.0 \pm 37.9 \% \mathrm{MVC}$ and 19.6 $\pm 11.0 \%$ MVC, respectively). In two cases, the opposite was true, which in Table 2 is indicated by a minus sign. In the countermovement phase the anterior deltoideus activity was slightly smaller $(p<$ $0.05)$ for the TS than for the CMJ $(12.6 \pm 9.7 \%$ MVC and $14.7 \pm 9.2 \% \mathrm{MVC}$, respectively).In the flight phasethe medial gastrocnemius activity was also slightly smaller $(p<0.05)$ for the TS jump than for the CMJ jump $(53.3 \pm 13.3 \%$ MVC and $73.7 \pm 34.8 \%$ MVC, respectively).

In the take-off phase of the TS and CMJ the average muscle activation levels of the rectus femoris and biceps femoris are particularly interesting (Table 2). In the TS, the EMG activity of the rectus femoris was significantly smaller $(\mathrm{p}<0.05)$ than in the CMJ $(21.7$ $\pm 26.5 \%$ MVC and $63.4 \pm 34.9 \%$ MVC, respectively). 
However, the biceps femoris activity in the TS was significantly higher $(p<0.05)$ than in the CMJ $(77.5$ $\pm 27.6 \%$ MVC and $38.9 \pm 23.6 \%$ MVC, respectively).

Tucked backward somersault vs. piked backward somersault. A comparison of EMG patterns in the set of muscles between the tucked and piked backward somersault performed by the same group of gymnasts found them to be very similar (Figures $2 \mathrm{~A}$ and 2B; Table 2). The Wilcoxon test demonstrated that the average muscle activation levels for all eight muscles in three subsequent phases between the TS and the CMJ did not differ statistically $(p>0.05)$.

Tucked backward somersault vs. backward handspring. As could be expected, statistically significant differences $(p<0.05)$ in average muscle activation levels were found only in the flight phase between the TS and the HS (Table 2). This is true for almost all muscles. However, only in the rectus femoris and rectus abdominis the average muscle activation levels were slightly higher $(p<0.05)$ for the TS in comparison to the HS (for RF $56.9 \pm 25.4 \%$ MVC and $14.6 \pm 11.9 \%$ MVC, respectively; for RA $76.0 \pm 37.9 \%$ MVC and $15.7 \pm 6.4 \% \mathrm{MVC}$, respectively). In contrast, the activity of other muscles, the biceps femoris, gluteus maximus, erector spinae, and anterior deltoideus, was significantly smaller $(p<0.05)$ for the TS than for the HS (for BF $40.0 \pm 12.8 \% \mathrm{MVC}$ and $61.4 \pm 18.8 \% \mathrm{MVC}$, respectively; for GM $16.0 \pm 5.4 \%$ MVC and $77.4 \pm 43.6 \%$ MVC, respectively; for ES $25.7 \pm 8.5 \%$ MVC and 109.1 $\pm 39.0 \%$ MVC, respectively; for AD $27.8 \pm 20.6 \%$ MVC and $81.8 \pm 38.5 \%$ MVC, respectively).

External structure of standing acrobatic jumps

External structures of the TS, CMJ, PS, and HS are shown in Figures 4-9. The external structure of acrobatic jumps consists of the kinetic structure i.e., the averaged and temporarily normalized vertical ground reaction force curve (Figures 4-6) and of the kinematic structure i.e., the averaged and temporarily normalized angle-time curves for the relative angles of upper and lower leg joints (Figures 7-9). A comparison of all temporal characteristics and the variables between acrobatic jumps (Table 3) was performed for the same group of gymnasts.

Force plate variables

In the take-off phase of the tucked somersault there was a significantly smaller $(p<0.05)$ vertical impulse $\left(F \cdot t_{y}\right)$ and the body's centre of mass had a significantly smaller $(p<0.05)$ vertical velocity $\left(v_{y}\right)$ at the instant of the take-off than in the countermovement jump (Table 3$)\left(F \cdot t_{y}=139.9 \pm 22.4 \mathrm{~N} \cdot \mathrm{s}\right.$ and $\quad F \cdot t_{y}=158.6 \pm 26.3 \mathrm{~N} \cdot \mathrm{s}$, respectively; $v_{y}=2.43 \pm 0.21 \mathrm{~m} / \mathrm{s}$ and $v_{y}=2.70 \pm 0.21 \mathrm{~m} / \mathrm{s}$, respectively).The body's centre of mass was also located lower to the ground. Vertical displacement of the COM $\left(d_{y}\right)$ in the flight phase for the TS in comparison to the CMJwas significantly smaller $(p<$ $0.05)\left(d_{y}=0.30 \pm 0.05 \mathrm{~m}\right.$ and $d_{y}=0.37 \pm 0.05 \mathrm{~m}$, respectively). However, the horizontal impulses $\left(F \cdot t_{x}\right)$ in the take-off phase for both the TS and the CMJ were almost the same $\left(F \cdot t_{x}=-0.4 \pm 8.8 \mathrm{~N} \cdot \mathrm{s}\right.$ and $F \cdot t_{x}=-1.4 \pm 7.2$ $\mathrm{N} \cdot \mathrm{s}$, respectively).

A comparison of the vertical impulses, velocities and displacements for the tucked and the piked backward somersaults demonstrated that the values of these variables in the TS were significantly higher $(p<0.05$; Table 3$)$ than in the PS jump $\left(F \cdot t_{y}=\right.$ $133.6 \pm 28.4 \mathrm{~N} \cdot \mathrm{s}$ and $F \cdot t_{y}=128.1 \pm 25.4 \mathrm{~N} \cdot \mathrm{s}$, respectively; $v_{y}=2.41 \pm 0.25 \mathrm{~m} / \mathrm{s}$ and $v_{y}=2.22 \pm 0.25 \mathrm{~m} / \mathrm{s}$, respectively; $d_{y}=0.30 \pm 0.06 \mathrm{~m}$ and $d_{y}=0.26 \pm 0.05 \mathrm{~m}$, respectively).The horizontal impulse in the take-off phase of the TS was significantly smaller than the PS $\left(F \cdot t_{x}=3.2 \pm 9.0 \mathrm{~N} \cdot \mathrm{s}\right.$ and $F \cdot t_{x}=-9.1 \pm 12.5 \mathrm{~N} \cdot \mathrm{s}$, respectively). However, only a statistical tendency was found $(p=0.0630)$.

The same must be said with regard to the tucked backward somersault in comparison to the backward handspring. The vertical impulses, velocities and displacements for the TS versus the HS were very significantly $(p<0.05)$ higher $\left(F \cdot t_{y}=135.5\right.$ $\pm 14.0 \mathrm{~N} \cdot \mathrm{s}$ and $F \cdot t_{y}=82.8 \pm 16.5 \mathrm{~N} \cdot \mathrm{s}$, respectively; $v_{y}=$ $2.42 \pm 0.14 \mathrm{~m} / \mathrm{s}$ and $v_{y}=1.48 \pm 0.31 \mathrm{~m} / \mathrm{s}$, respectively; $d_{y}$ $=0.30 \pm 0.04 \mathrm{~m}$ and $d_{y}=0.11 \pm 0.05 \mathrm{~m}$, respectively). However, the horizontal impulses in the take-off phase between the TS and the HS were significantly $(p$ $<0.05)$ smaller $\left(F \cdot t_{x}=-2.8 \pm 7.7 \mathrm{~N} \cdot \mathrm{s}\right.$ and $F \cdot t_{x}=-22.1 \pm 10.6$ $\mathrm{N} \cdot \mathrm{s}$, respectively).

Variables from infrared cameras

The vertical speeds and displacements calculated from data obtained from infrared cameras which compared a tucked somersault with three other acrobatic jumps are similar to those from the force plate. The highest magnitude of vertical velocity and displacement of COM was observed in the countermovement jump, followed by the tucked somersault, piked somersault, and handspring $\left(v_{y}=\right.$ $2.67 \pm 0.18 \mathrm{~m} / \mathrm{s}, v_{y}=2.32 \pm 0.21 \mathrm{~m} / \mathrm{s}, v_{y}=2.21 \pm 0.28 \mathrm{~m} / \mathrm{s}$, and no data for technical reason, respectively, $d_{y}=0.37$ $\pm 0.05 \mathrm{~m}, d_{y}=0.28 \pm 0.05 \mathrm{~m}, d_{y}=0.25 \pm 0.06 \mathrm{~m}$, and no data for technical reason, respectively). The Wilcoxon test demonstrated that both the vertical velocity and displacement of the COM between the TS and the CMJ and between the TS and the PS were statistically 
different $(p<0.05$; Table 3$)$.

The moment of inertia (I), the angular velocity $(\omega)$ and the angular momentum $(H)$ for the TS compared to the PS were significantly $(p<0.05)$ smaller $\left(I=12.08 \pm 1.64 \mathrm{~kg} \cdot \mathrm{m}^{2}\right.$ and $I=12.23 \pm 1.50$ $\mathrm{kg} \cdot \mathrm{m}^{2}$, respectively; $\omega=4.38 \pm 0.30 \mathrm{rad} / \mathrm{s}$ and $\omega=$ $5.75 \pm 0.39 \mathrm{rad} / \mathrm{s}$, respectively; $H=53.1 \pm 8.2 \mathrm{~kg} \cdot \mathrm{m}^{2} / \mathrm{s}$ and $H=67.9 \pm 6.9 \mathrm{~kg} \cdot \mathrm{m}^{2} / \mathrm{s}$, respectively). When the TS jumps were compared with the HS jumps, the opposite was true $(p<0.05)(\omega=4.43 \pm 0.55 \mathrm{rad} / \mathrm{s}$ and $\omega=6.20 \pm 0.93 \mathrm{rad} / \mathrm{s}$, respectively; $H=55.2 \pm 13.0$ $\mathrm{kg} \cdot \mathrm{m}^{2} / \mathrm{s}$ and $H=70.9 \pm 14.7 \mathrm{~kg} \cdot \mathrm{m}^{2} / \mathrm{s}$, respectively). In Table 3 this is indicated by a minus sign. The only exception was the moment of inertia, which in the backward somersault was slightly higher $(p<0.05)$ at the instant of the take-off than in the backward handspring $\left(I=12.35 \pm 1.60 \mathrm{~kg} \cdot \mathrm{m}^{2}\right.$ and $I=11.46 \pm 1.79$ $\mathrm{kg} \cdot \mathrm{m}^{2}$, respectively).

\section{Discussion and practical implications}

This study presents the phase structure of the movement and is focused on the variables that can primarily affect the take-off by comparing four different acrobatic jumps. A large number of studies have focused on the biomechanical and neurophysiological variables involved in vertical jumps (Jacobs et al., 1996; Pereira et al., 2008; van Soest et al., 1993; Wikstrom et al., 2008; van Zandwijk et al., 2000) and their influence on performance. However, significantly fewer studies have been done on acrobatic jumps (McNeal et al., 2007; Mkaouer et al., 2012, 2014; Okubo, 2012).

Bearing in mind that biomechanics is concentrated on forces that act on a human body and the effects of these forces, the first thing to be considered is the muscle action. Skeletal muscles are the primary initiator of movement and are a real biological system designed to produce mechanical force and induce movement. Knowledge of the characteristics of muscle activation during the subsequent phases of the movement may enhance our understanding of how gymnasts perform these basic acrobatic jumps and may assist coaches and physical educators in prescribing appropriate training drills for particular jumps.

Studying the muscle activity of eight muscles in the three successive phases (the countermovement, take-off, and flight phase) of four standing 'acrobatic jumps'(TS, PS, HS and CMJ) it can be stated that in most cases, the highest average muscle activation levels of these muscles were found during the take-off phase (Figures 1-3). The exceptions were the rectus femoris and the rectus abdominis, which in the tucked and piked backward somersault had the highest values in the flight phase. The difference of the rectus abdominis activity in the flight phase, between the TS and the CMJ was 56.4\% MVC, which, with standard deviations, \pm 37.9 and $\pm 11.0 \%$ MVC, respectively, was significant ( $p=0.0033$; Table. 2$)$. A similar regularity was also found for the rectus femoris activity ( $p=$ $0.0164)$. The same phenomenon in the flight phase was observed in the rectus abdominis and the rectus femoris by Okubo (2012), who found that greater muscular activity was present during a tucked backward somersault than in a vertical jump. This is understandable due to rapid flexing in the hip joints during the flight phase of somersaults.

Jacobs et al. (1996) proposed that biarticular muscles were very important for jumping. These authors found that the rectus femoris transferred power from the hip to the knee. In contrast the kinematic analysis during vertical jumps showed that hamstrings were involved in the power transfer from knees to the hip. The result of the simultaneous contraction of these antagonist muscles is the net transfer of force from the hip to the knees. Taking this into consideration, the current findings are especially interesting. In the take-off phase of acrobatic jumps with rotation (TS, PS and HS) the average muscle activation levels of biarticular biceps femoris were significantly higher $(p<0.05)$ and biarticular rectus femoris significantly lower $(p<0.05)$ than in the countermovement jump. This means that these muscles have different roles. In the TS, PS and HS the role of the rectus femoris muscle changes from a prime mover to a supportive-prime mover (a stabilizing muscle). Conversely in the CMJ, the rectus femoris acts as a prime mover and the biceps femoris acts as a stabilizing muscle. Therefore, electromyography is a dynamic type of assessment for neuromuscular control, which quantifies preparatory and reflexive muscle amplitudes around a particular joint, which is an important indicator in determining joint stability during functional tasks (Ebig et al., 1997; McKinley and Pedotti, 1992; Pereira et al., 2008; Wikstrom et al., 2008). However, an equally important indicator of muscle activation is the possibility of movement in the joint. 
Table 1

Descriptive characteristics of participants.

\begin{tabular}{ccccc}
\hline & Age [years] & Body height $[\mathrm{m}]$ & Body mass [kg] & $\begin{array}{c}\text { Training } \\
\text { experience [years] }\end{array}$ \\
\cline { 2 - 5 } Mean $( \pm \mathrm{SD})$ & $16.4(1.4)$ & $1.64(0.05)$ & $57.8(6.3)$ & $10.5(2.07)$ \\
Range & $15-19$ & $1.54-1.70$ & $45.6-68.3$ & $8-14$ \\
\hline
\end{tabular}

Table 2

Comparative statistics of four standing 'acrobatic' jumps (tucked somersault - TS, countermovement jump - CMJ, piked somersault - PS, Handspring - HS) in successive phases of the movement. Variables - RMS EMG activity from the eight muscles. A comparison of all variables (temporal characteristics) between acrobatic jumps was performed

for the same group of gymnasts.

\begin{tabular}{|c|c|c|c|c|c|c|c|c|c|}
\hline \multirow[t]{2}{*}{ Variables } & \multicolumn{9}{|c|}{ Wilcoxon Signed-Ranks Test } \\
\hline & \multicolumn{3}{|c|}{ TS vs. CMJ } & \multicolumn{3}{|c|}{ TS vs. PS } & \multicolumn{3}{|c|}{ TS vs. HS } \\
\hline & \multicolumn{9}{|c|}{ Countermovement phase } \\
\hline & $N$ & $T$ & $p$ & $N$ & $T$ & $p$ & $N$ & $T$ & $p$ \\
\hline Anterior tibialis & 11 & 22 & 0.3281 & 7 & 5 & 0.1282 & 8 & 18 & 1.0000 \\
\hline Medial gastrocnemius & 11 & 7 & $0.0208^{*}$ & 7 & 9 & 0.3980 & 8 & 8 & 0.1614 \\
\hline Rectus femoris & 11 & 0 & $0.0033^{*}$ & 7 & 4 & 0.0910 & 8 & 11 & 0.3270 \\
\hline Biceps femoris & 11 & 1 & $0.0044^{*}$ & 7 & 12 & 0.7353 & 8 & 17 & 0.8886 \\
\hline Rectus abdominis & 11 & 8 & $0.0262^{*}$ & 7 & 10 & 0.4990 & 8 & 6 & 0.0929 \\
\hline Gluteus maximus & 11 & 11 & 0.0546 & 7 & 6 & 0.1763 & 8 & 17 & 0.8886 \\
\hline Erector spinae & 11 & 20 & 0.2477 & 7 & 10 & 0.4990 & 8 & 8 & 0.1614 \\
\hline \multirow[t]{3}{*}{ Anterior deltoideus } & 11 & -7 & $0.0208^{*}$ & 7 & 10 & 0.4990 & 8 & 8 & 0.1614 \\
\hline & \multicolumn{9}{|c|}{ Take-off phase } \\
\hline & $N$ & $T$ & $p$ & $N$ & $T$ & $p$ & $N$ & $T$ & $p$ \\
\hline Anterior tibialis & 11 & 28 & 0.6566 & 7 & 4 & 0.0910 & 8 & 7 & 0.1235 \\
\hline Medial gastrocnemius & 11 & 24 & 0.4236 & 7 & 9 & 0.3980 & 8 & 5 & 0.0687 \\
\hline Rectus femoris & 11 & -0 & $0.0033^{*}$ & 7 & 3 & 0.0630 & 8 & 10 & 0.2626 \\
\hline Biceps femoris & 11 & 0 & $0.0033^{*}$ & 7 & 6 & 0.1763 & 8 & 4 & 0.0500 \\
\hline Rectus abdominis & 11 & 20 & 0.2477 & 7 & 11 & 0.6121 & 8 & 8 & 0.1614 \\
\hline Gluteus maximus & 11 & 10 & 0.1307 & 7 & 3 & 0.0630 & 8 & 12 & 0.4008 \\
\hline Erector spinae & 11 & 32 & 0.9292 & 7 & 11 & 0.6121 & 8 & 8 & 0.1614 \\
\hline \multirow[t]{3}{*}{ Anterior deltoideus } & 11 & 11 & 0.0505 & 7 & 11 & 0.6121 & 8 & 10 & 0.2626 \\
\hline & \multicolumn{9}{|c|}{ Flight phase } \\
\hline & $N$ & $T$ & $p$ & $N$ & $T$ & $p$ & $N$ & $T$ & $p$ \\
\hline Anterior tibialis & 11 & 1 & $0.0044^{*}$ & 7 & 6 & 0.1763 & 8 & 2 & $0.0251^{*}$ \\
\hline Medial gastrocnemius & 11 & -7 & $0.0208^{*}$ & 7 & 10 & 0.4990 & 8 & 5 & 0.0687 \\
\hline Rectus femoris & 11 & 6 & $0.0164^{*}$ & 7 & 3 & 0.0630 & 8 & 1 & $0.0173^{*}$ \\
\hline Biceps femoris & 11 & 0 & $0.0033^{*}$ & 7 & 3 & 0.0630 & 8 & -1 & $0.0173^{*}$ \\
\hline Rectus abdominis & 11 & 0 & $0.0033^{*}$ & 7 & 10 & 0.4990 & 8 & 0 & $0.0117^{*}$ \\
\hline Gluteus maximus & 11 & 23 & 0.3739 & 7 & 7 & 0.2367 & 8 & -1 & $0.0173^{*}$ \\
\hline Erector spinae & 11 & 24 & 0.4236 & 7 & 9 & 0.3980 & 8 & -0 & $0.0117^{*}$ \\
\hline Anterior deltoideus & 11 & 18 & 0.1823 & 7 & 13 & 0.8658 & 8 & -0 & $0.0117^{*}$ \\
\hline
\end{tabular}

"-" The minus sign indicates that in the second of the compared jumps the average muscle activation level in the specified phase was significantly higher;

$N$ - number of valid sample pairs; ${ }^{*}$ - significance at $p<0.05$. 
Table 3

Comparative statistics of kinetic and kinematic variables of standing 'acrobatic' jumps (tucked somersault - TS; countermovement jump - CMJ; piked somersault - PS;

Handspring - HS) from a force plate and the infrared cameras.

Variables of all 'acrobatic' jumps were compared for the same group of gymnasts.

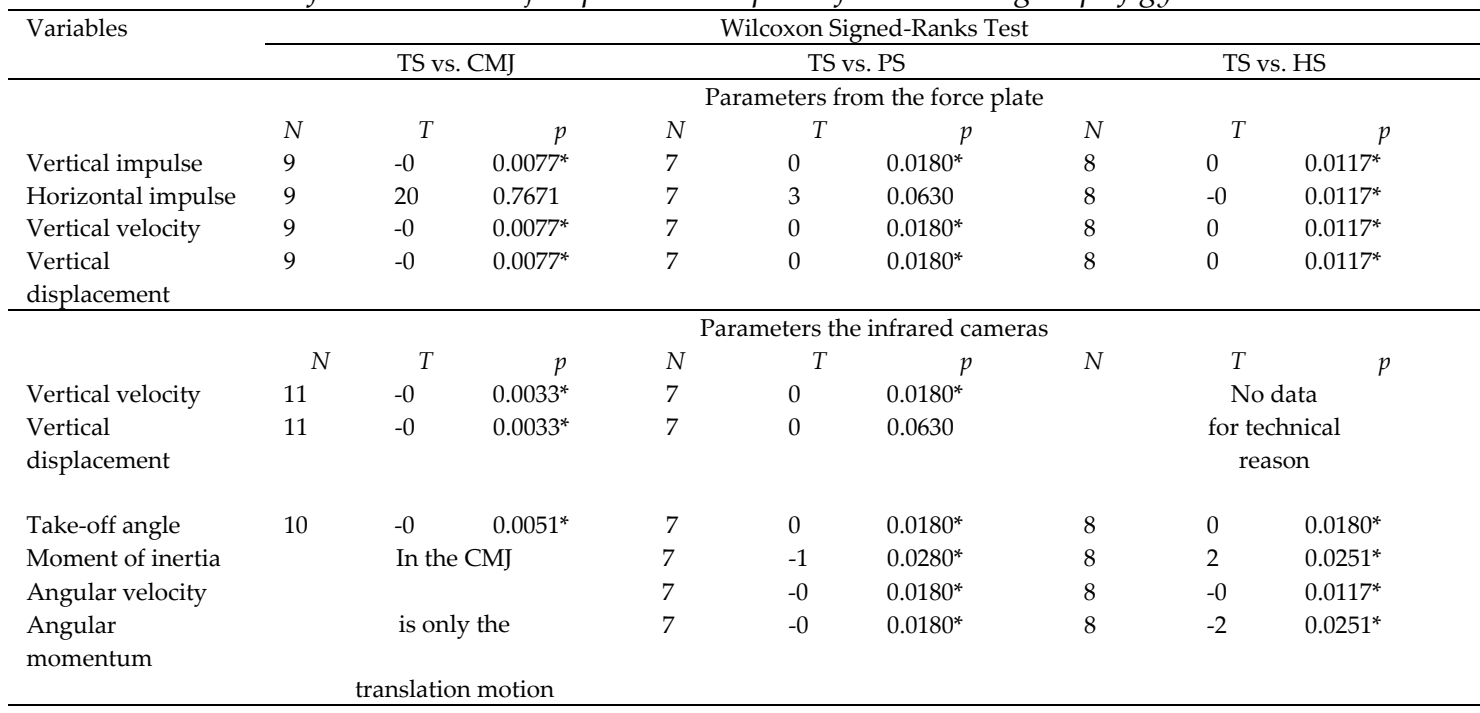

"-" The minus sign indicates that the value of this parameter in the second of the compared jumps was significantly greater; $N$ - number of valid sample pairs; ${ }^{*}$ - significance at $p<0.05$.

Table 4

The take-off angle $\left[^{\circ}\right]$ and anormalized RMS EMG[\% of MVC] average muscle activation level during the take-off phase of selected muscles for standing 'acrobatic' jumps (countermovement jump - CMJ; tucked somersault - TS;

\begin{tabular}{|c|c|c|c|c|c|c|c|c|}
\hline \multirow[t]{2}{*}{ Variables } & \multicolumn{2}{|r|}{ CMJ } & \multicolumn{3}{|l|}{ TS } & PS & \multicolumn{2}{|l|}{$\mathrm{H}$} \\
\hline & $\mathrm{N}$ & Mean SD & $\mathrm{N}$ & Mean SD & $\mathrm{N}$ & Mean SD & $\mathrm{N}$ & Mean SD \\
\hline Take-off angle & 11 & $91.5 \pm 1.5$ & 11 & $84.3 \pm 2.1$ & 7 & $79.6 \pm 1.7$ & 8 & $71.2 \pm 3.8$ \\
\hline Gastrocnemius & 11 & $159.4 \pm 68.7$ & 11 & $165.7 \pm 64.0$ & 7 & $139.7 \pm 41.8$ & 8 & $145.4 \pm 51.0$ \\
\hline Biceps femoris & 11 & $38.9 \pm 23.6$ & 11 & $77.5 \pm 27.6$ & 7 & $88.8 \pm 26.3$ & 8 & $89.6 \pm 32.4$ \\
\hline Rectus femoris & 11 & $63.4 \pm 34.9$ & 11 & $21.7 \pm 26.5$ & 7 & $13.2 \pm 5.6$ & 8 & $14.8 \pm 13.7$ \\
\hline Erector spinae & 11 & $111.2 \pm 62.7$ & 11 & $137.5 \pm 50.9$ & 7 & $144.5 \pm 46.2$ & 8 & $147.5 \pm 51.9$ \\
\hline
\end{tabular}

Table 5

The phase duration [s] for three subsequent phases of the standing 'acrobatic' jumps (countermovement jump-CMJ, tucked somersault - TS, piked somersault-PS, handspring - HS).

\begin{tabular}{|c|c|c|c|c|c|c|c|c|}
\hline \multirow[t]{2}{*}{ Phase } & \multicolumn{2}{|r|}{$\mathrm{CMJ}$} & \multicolumn{2}{|r|}{ TS } & \multicolumn{2}{|r|}{ PS } & \multicolumn{2}{|r|}{$\mathrm{H}$} \\
\hline & $\mathrm{N}$ & Mean SD & $\mathrm{N}$ & Mean SD & $\mathrm{N}$ & Mean SD & $\mathrm{N}$ & Mean SD \\
\hline Counter-movement & 11 & $1.16 \pm 0.28$ & 11 & $0.92 \pm 0.19$ & 7 & $0.82 \pm 0.19$ & 8 & $1.13 \pm 0.23$ \\
\hline Take-off & 11 & $0.30 \pm 0.07$ & 11 & $0.33 \pm 0.05$ & 7 & $0.32 \pm 0.06$ & 8 & $0.37 \pm 0.05$ \\
\hline Flight & 11 & $0.56 \pm 0.03$ & 11 & $0.61 \pm 0.03$ & 7 & $0.59 \pm 0.03$ & 8 & $0.32 \pm 0.05$ \\
\hline
\end{tabular}


a. Tucked somersault

b. Countermovement jump

Anterior tibialis
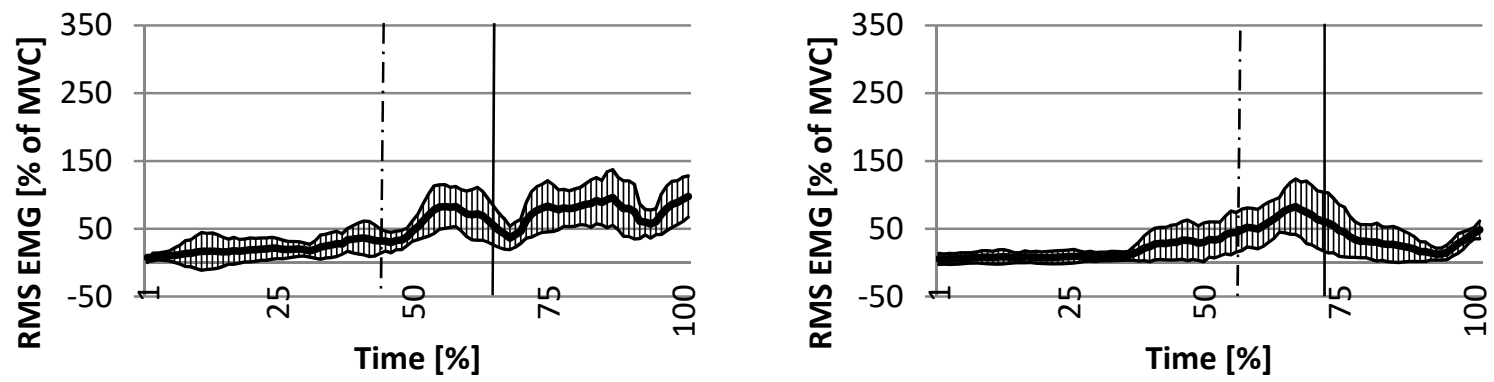

Medial gastrocnemius
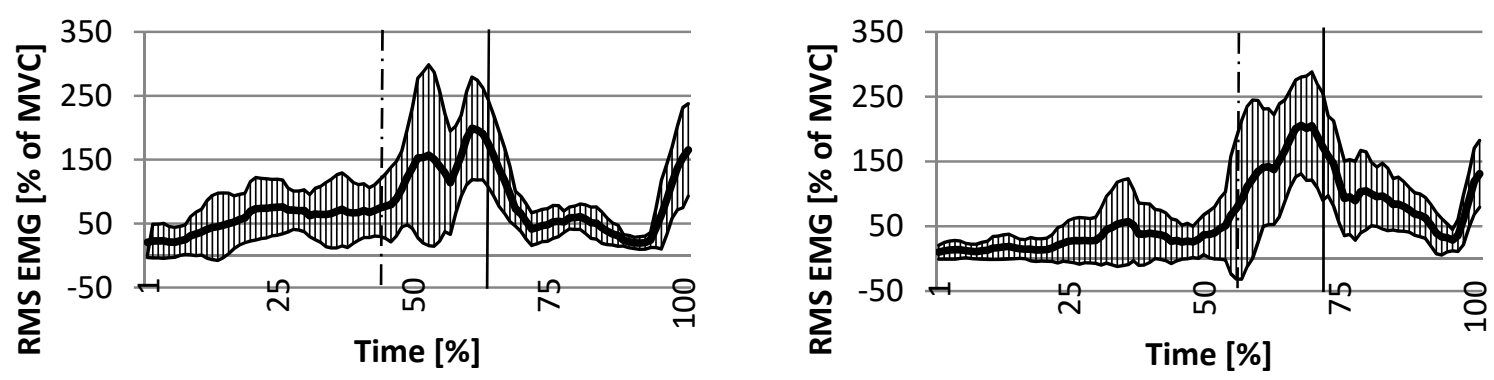

Rectus femoris
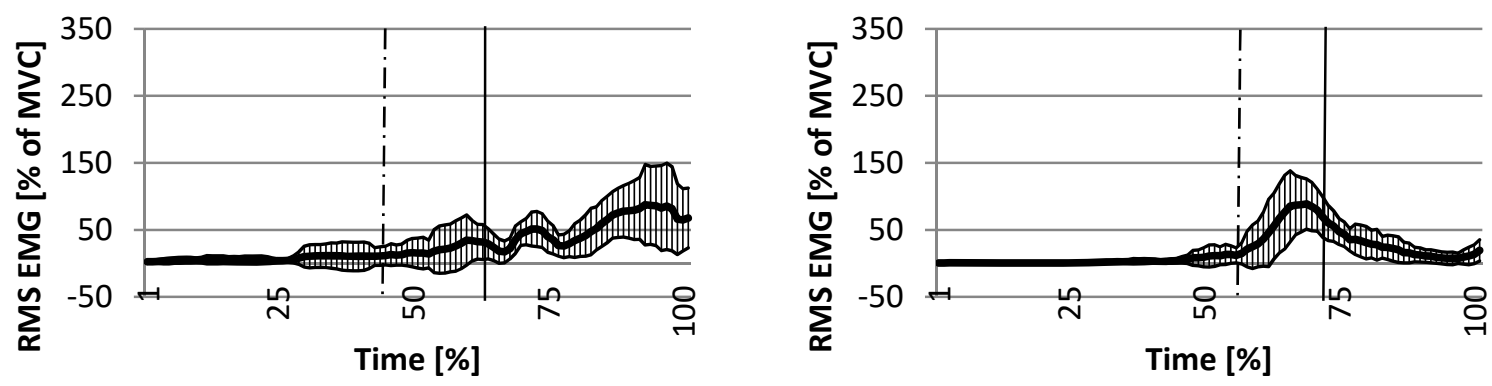

Biceps femoris
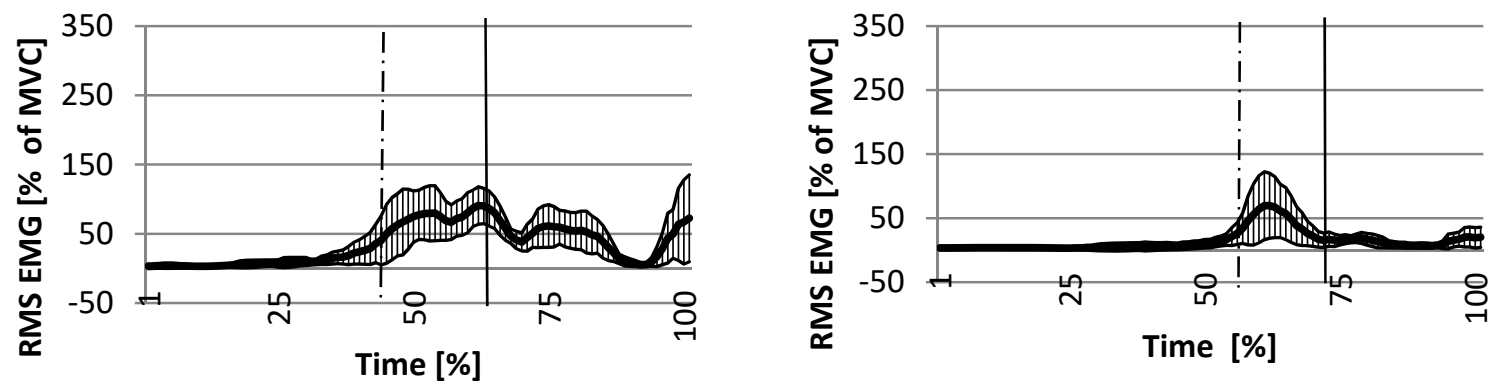

Figure 1. (A) Internal structure of the movement - the temporarily normalized RMS EMG $[\%$ of $M V C]$ linear envelopes (mean and $\pm S D$; thick and thin lines respectively) for four muscles during the standing tucked backward somersault (a) and the countermovement jump (b). All characteristics shown in Figures 1-9 have been adjusted to the instant of take-off (solid vertical line), while the dashed vertical line shows the transition from countermovement to the take-off phase. 
a. Tucked somersault

Rectus abdominis

b. Countermovement jump
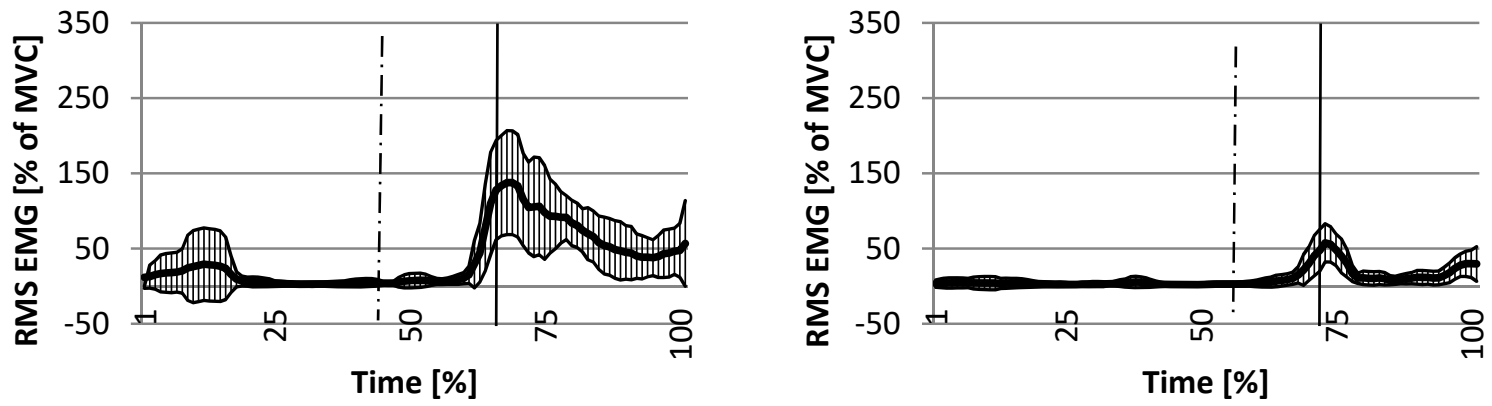

Gluteus maximus
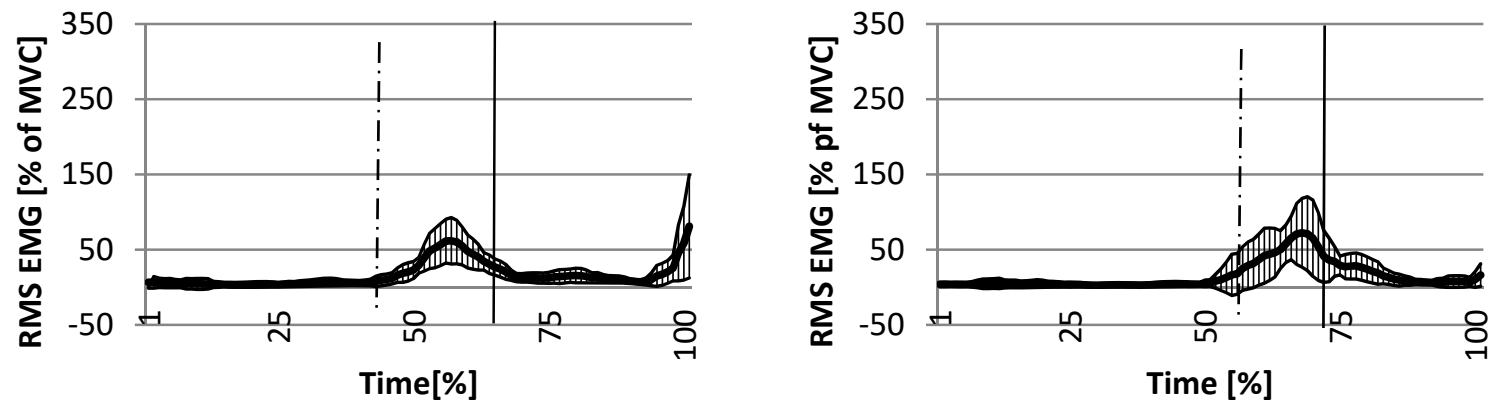

Erector spinae
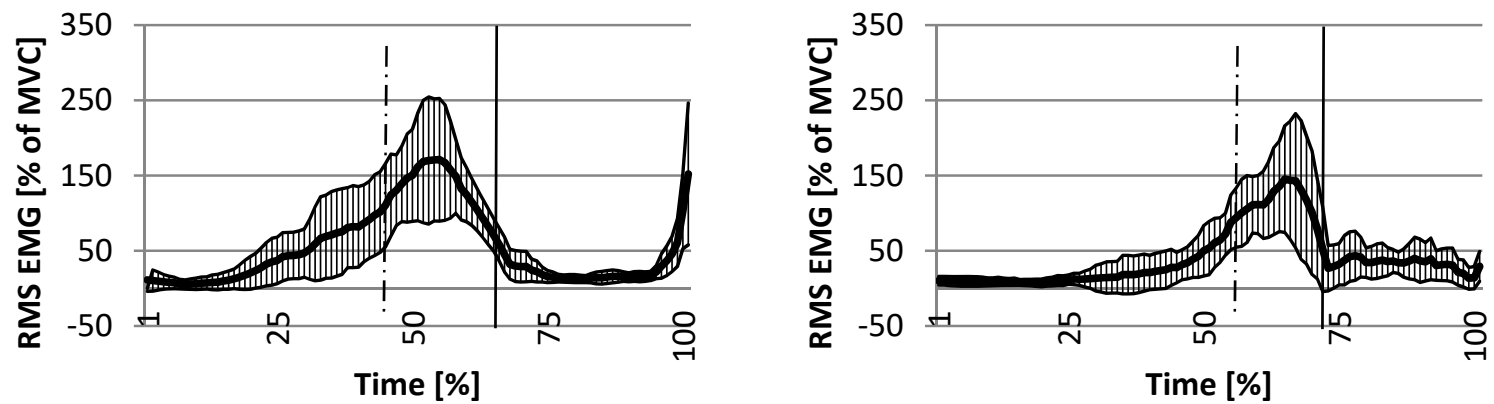

Anterior deltoideus
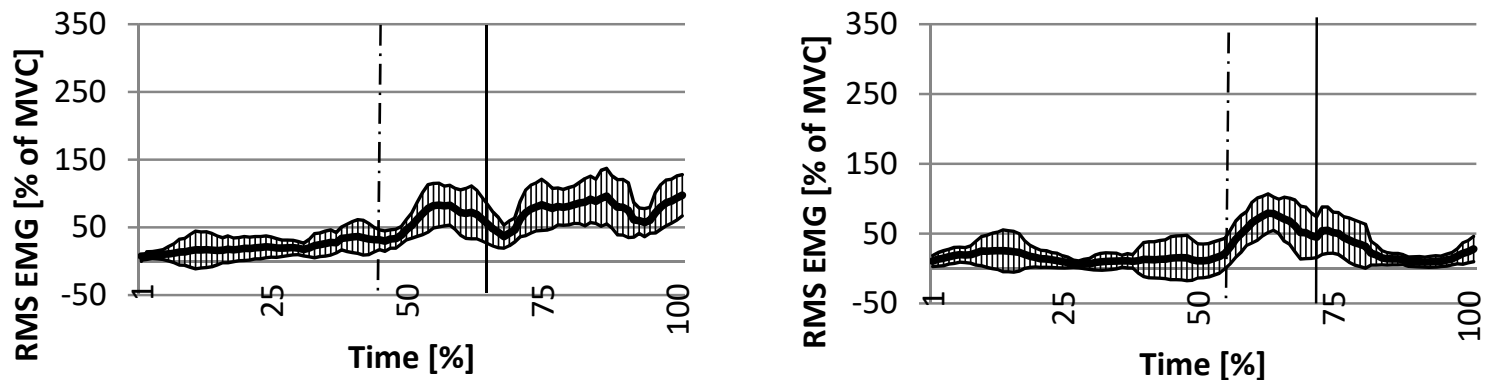

Figure 1. - (continued); (B) internal structure of the movement - the temporarily normalized RMS $E M G[\%$ of $M V C]$ linear envelopes for four remaining muscles during the standing tucked backward somersault (a) and the countermovement jump (b). 

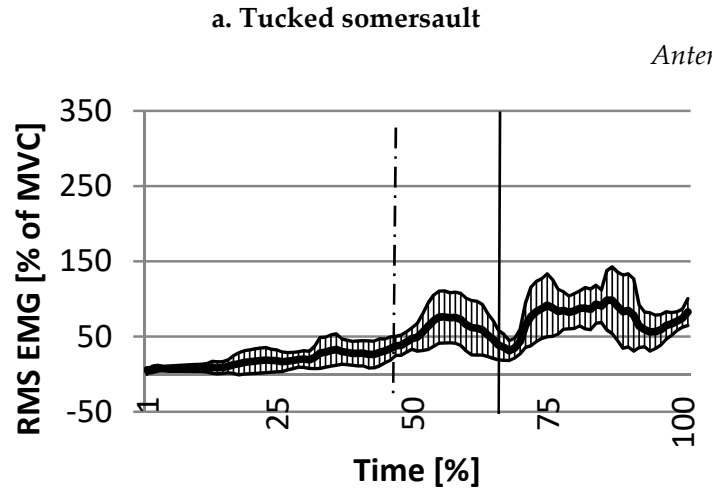

b. Piked somersault

Anterior tibialis

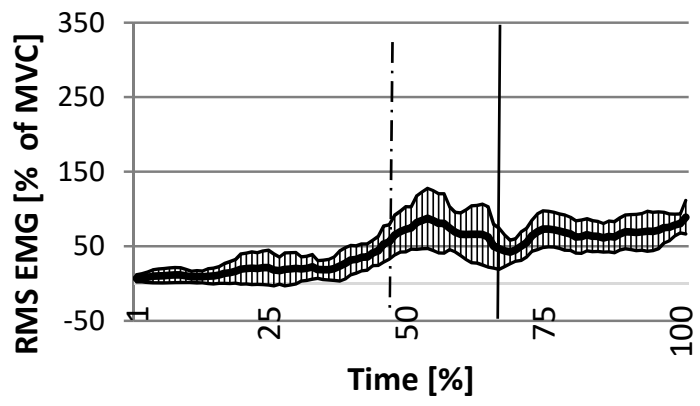

Medial gastrocnemius
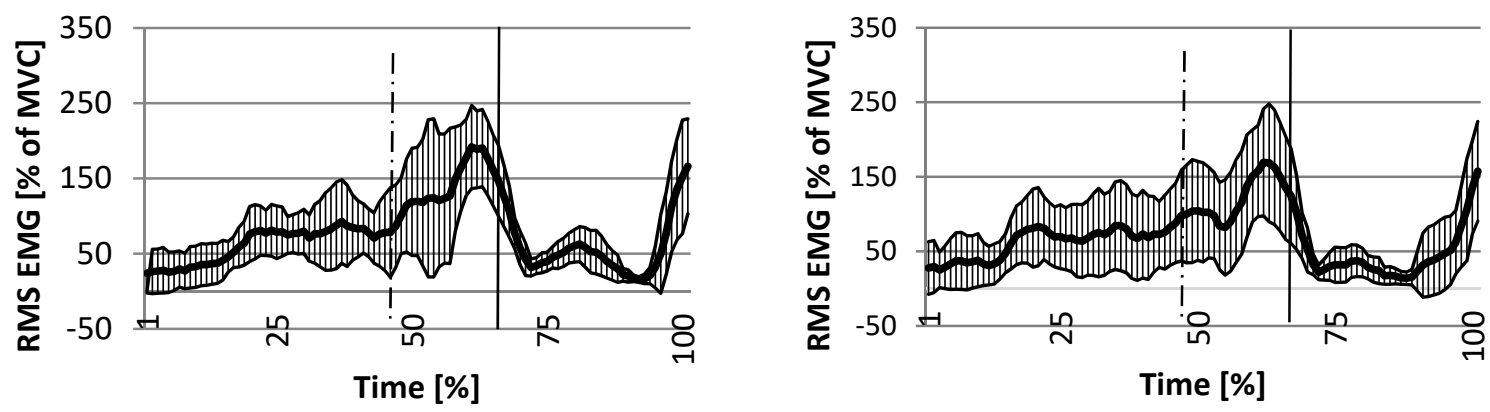

Rectus femoris
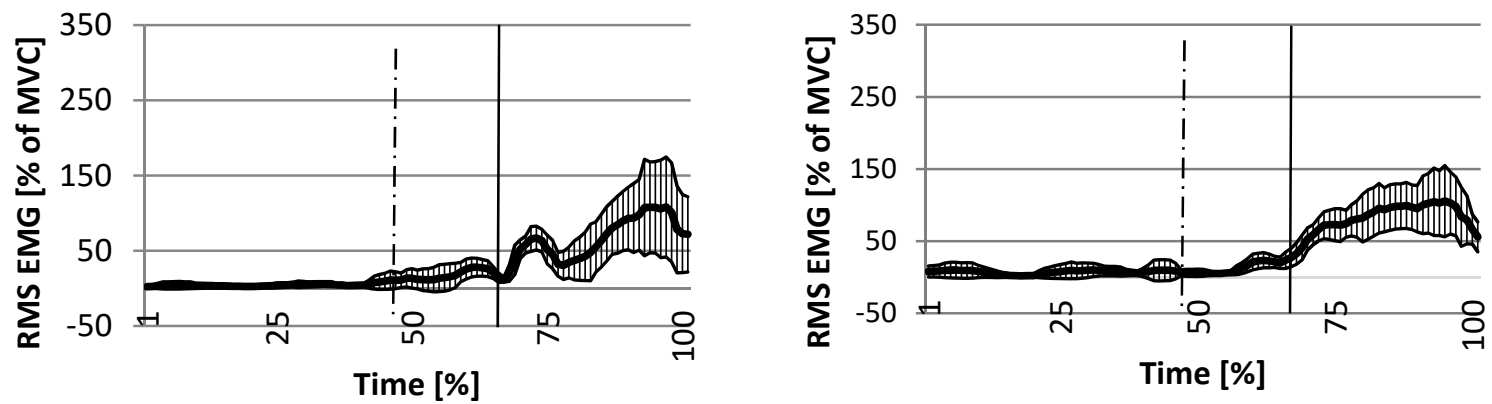

Biceps femoris
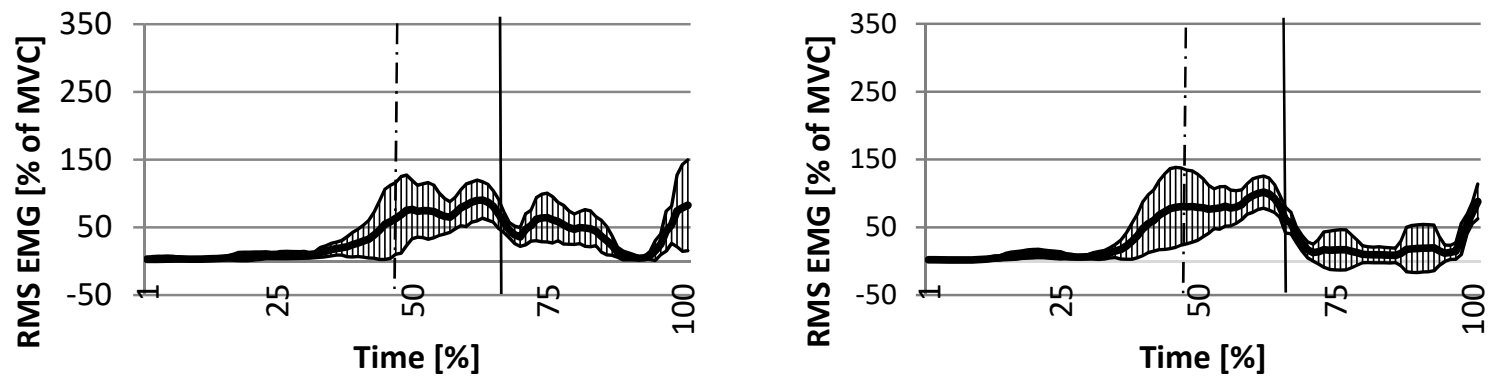

Figure 2. (A) Internal structure of the movement - the temporarily normalized RMS EMG $[\%$ of $M V C]$ linear envelopes (mean and $\pm S D$; thick and thin lines respectively) for four muscles during the standing tucked backward somersault (a) and the standing piked backward somersault (b). Further explanations as in Figure 1. 
a. Tucked somersault

b. Piked somersault

Rectus abdominis
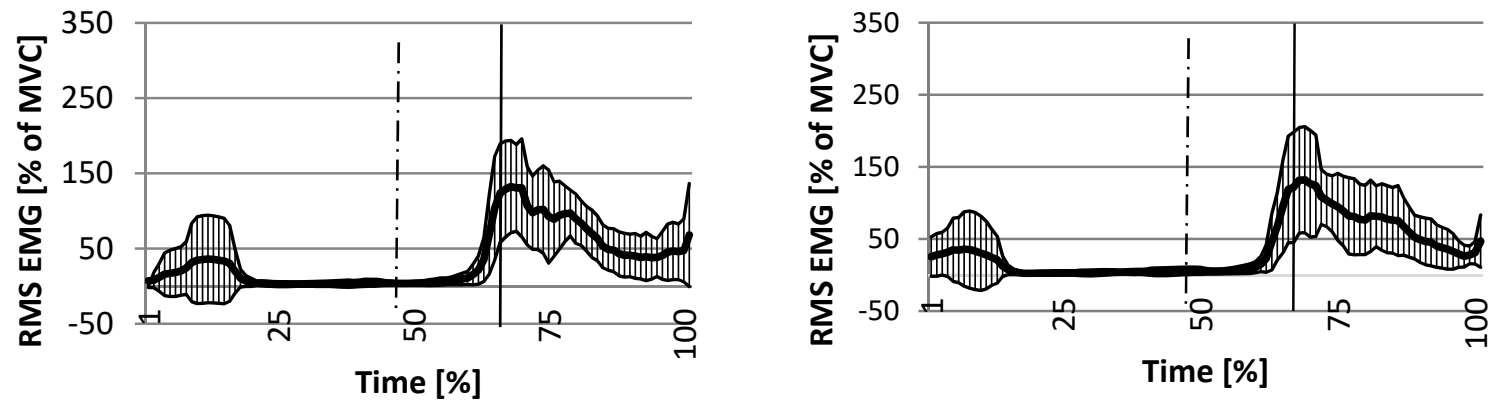

Gluteus maximus
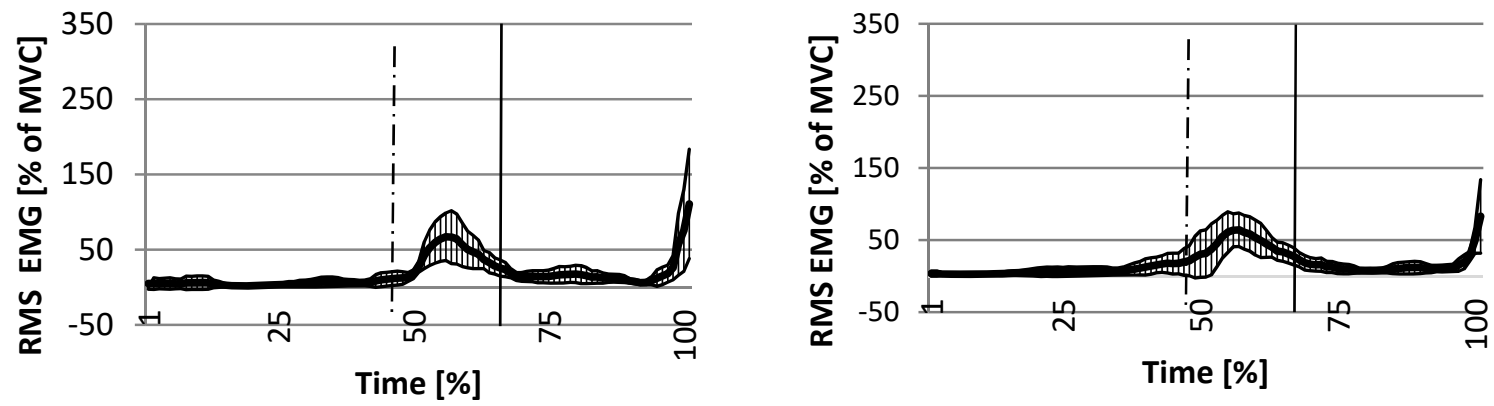

Erector spinae
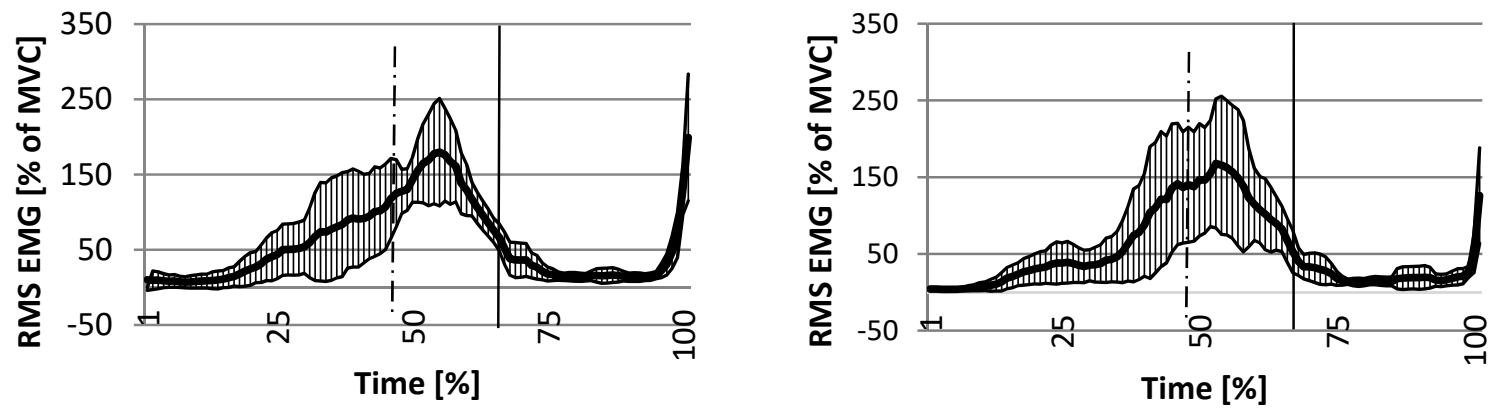

Anterior deltoideus
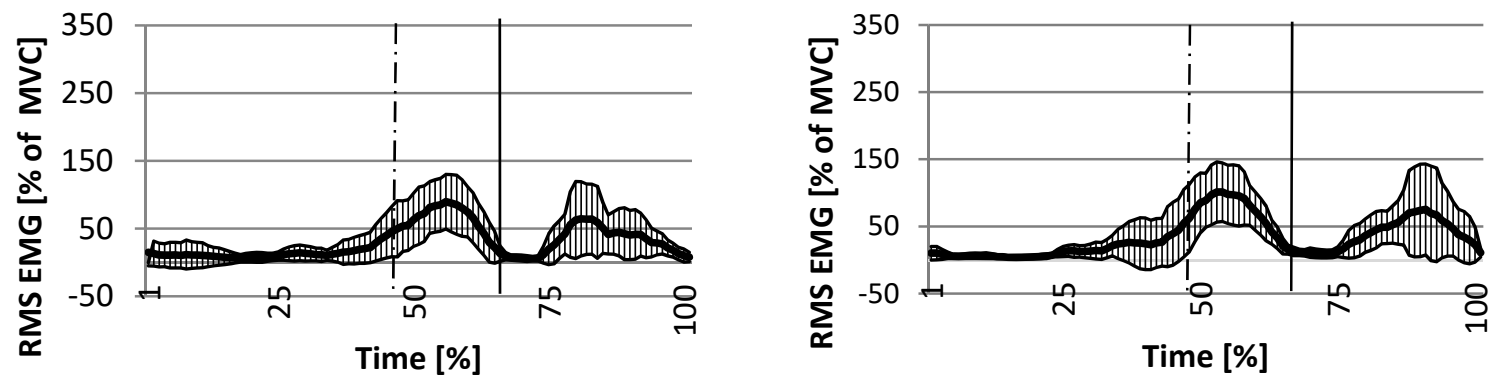

Figure 2.-(continued); (B)internal structure of the movement- the temporarily normalized RMS EMG [\% of MVC] linear envelopes for four remaining muscles during the standing tucked backward somersault ( $a$ ) and the standing piked backward somersault (b). 
a. Tucked somersault

Anterior tibialis

b. Handspring

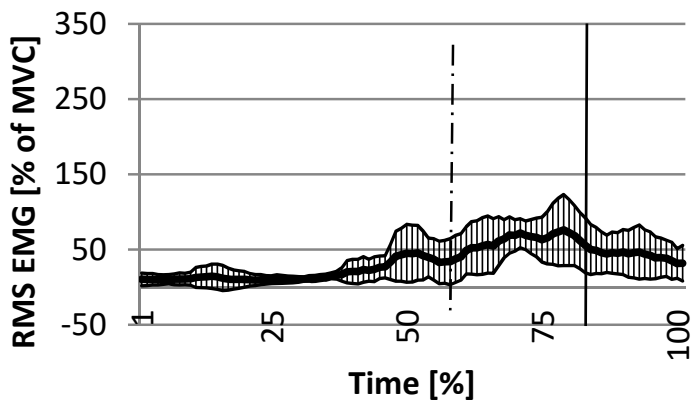

Medial gastrocnemius
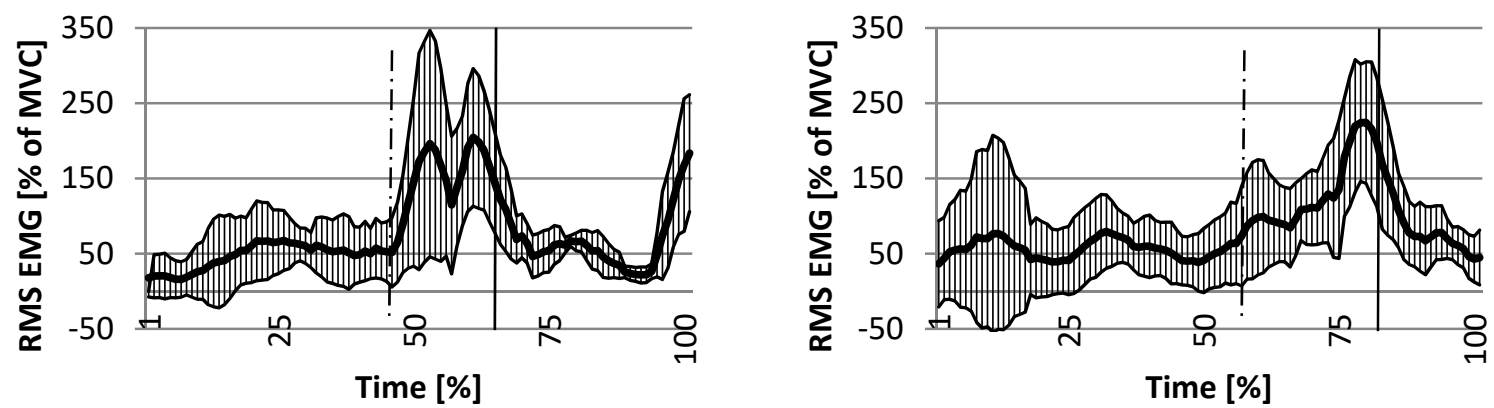

Rectus femoris
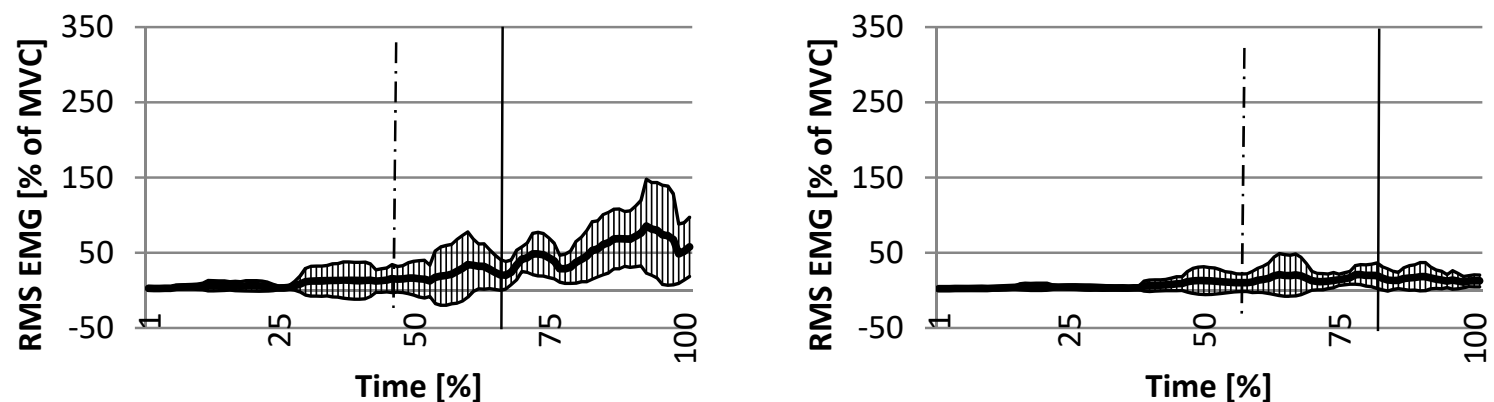

Biceps femoris
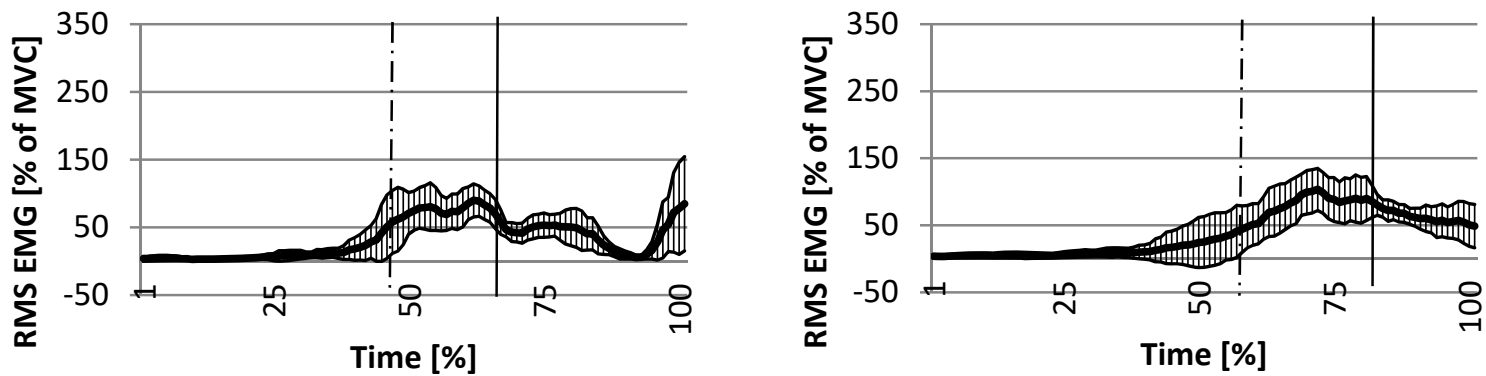

Figure 3. (A) Internal structure of the movement - the temporarily normalized RMS EMG $[\%$ of MVC] linear envelopes (mean and $\pm S D$; thick and thin lines respectively) for four muscles during the standing tucked backward somersault (a) and the standing backward handspring (b). Further explanations as in Figure 1. 
a. Tucked somersoult

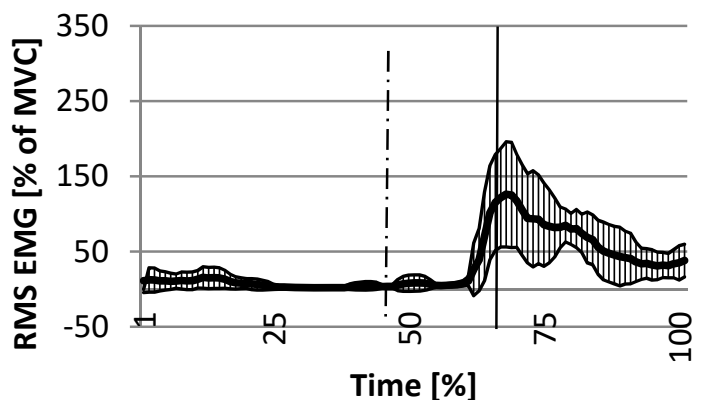

b. Handspring

Rectus abdominis

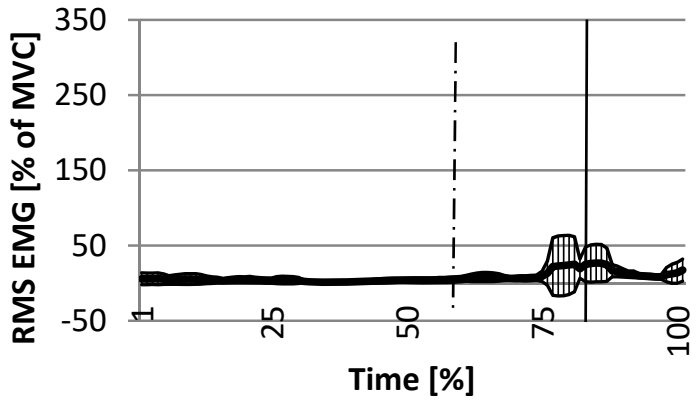

Gluteus maximus
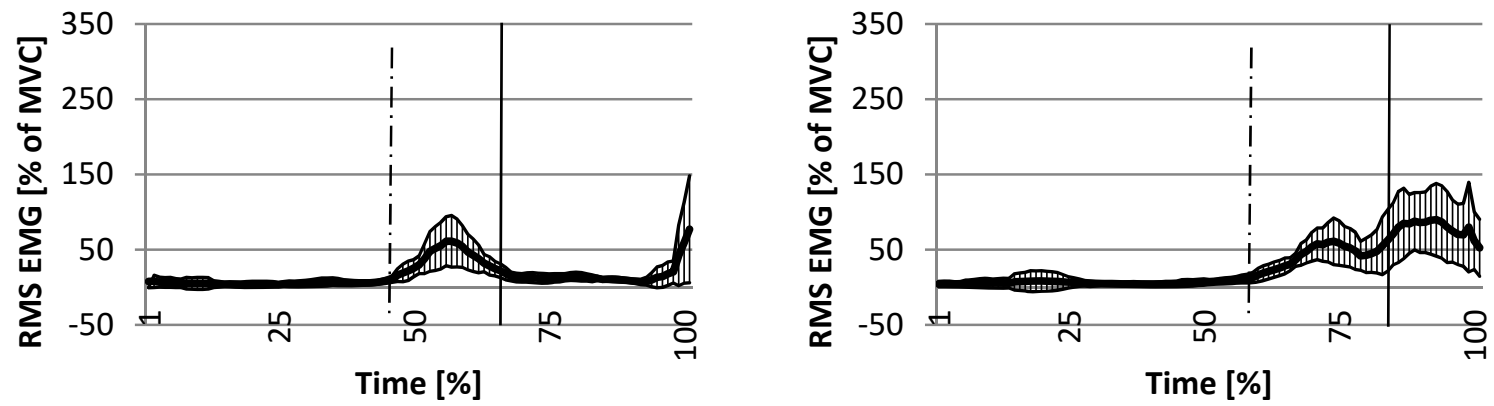

Erector spinae
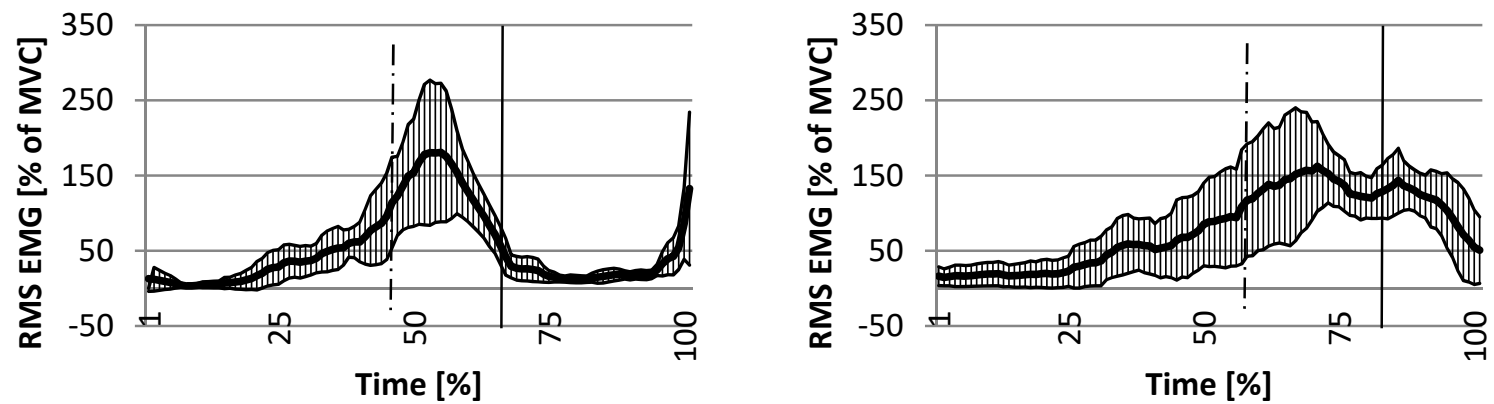

Anterior deltoideus
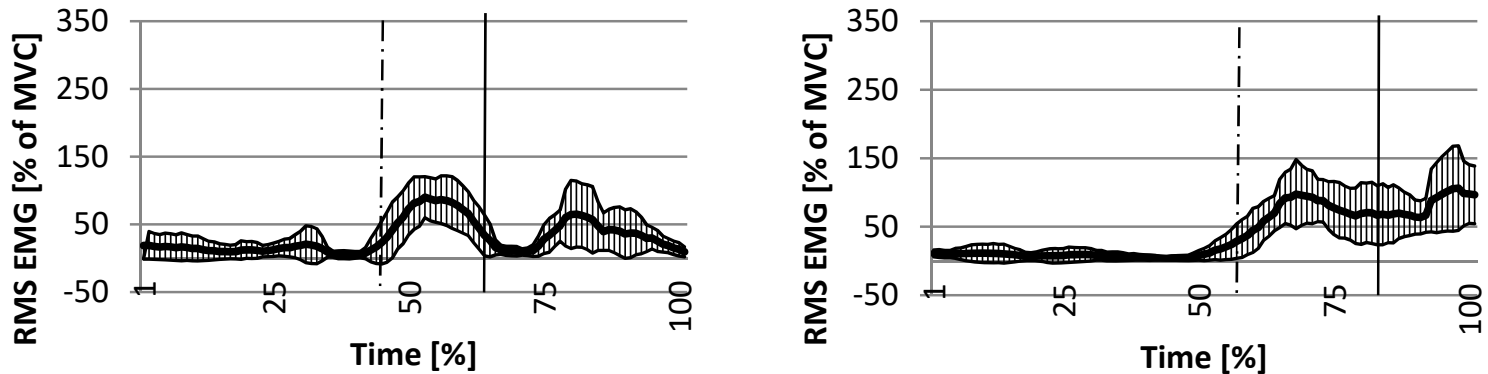

Figure 3.- (continued); (B) internal structure of the movement- the temporarily normalized RMS $E M G[\%$ of MVC] linear envelopes for four remaining muscles during the standing tucked backward somersault (a) and the standing backward handspring (b). 


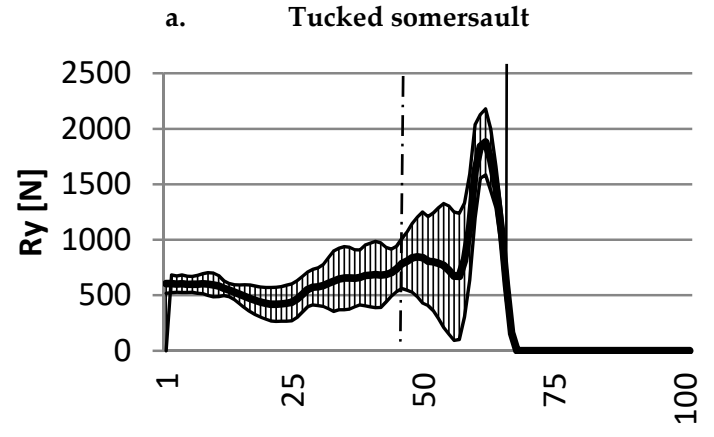

Time [\%]

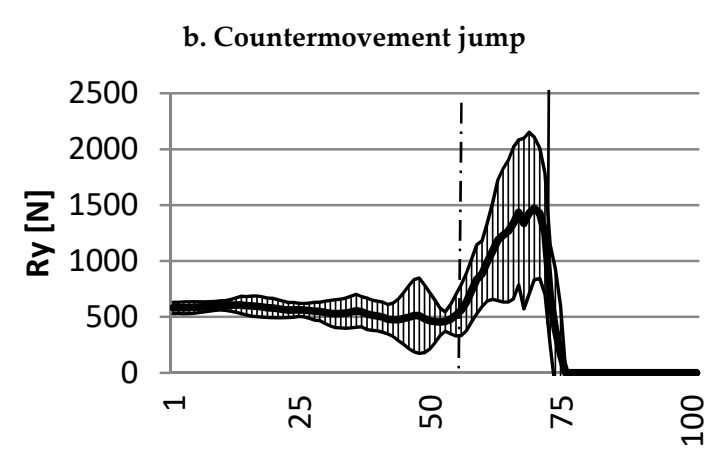

Time [\%]

Figure 4. External-kinetic structure of the movement - the averaged and temporarily normalized vertical ground reaction force $-R_{y}[N]$ during the standing tucked backward somersault (a) and countermovement jump (b). Further explanations as in Figure 1.

a. Tucked somersault

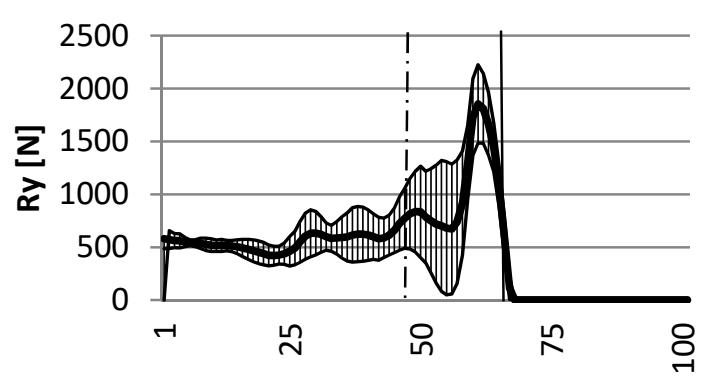

Time [\%] b. Piked somersault

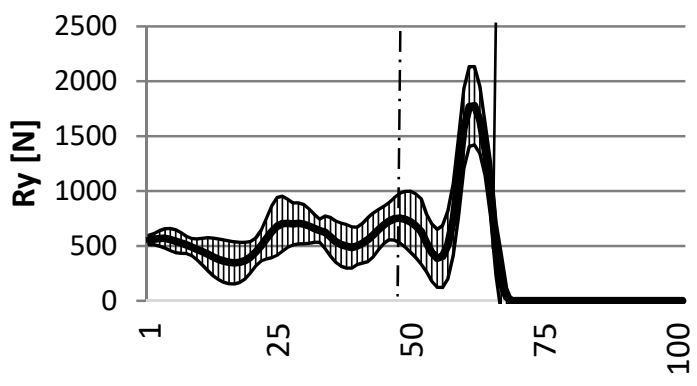

Time [\%]

Figure 5. External-kinetic structure of the movement - the averaged and temporarily normalized vertical ground reaction force $-R_{y}[N]$ during the standing tucked backward somersault (a) and the standing piked backward somersault (b). Further explanations as in Figure 1.

a.

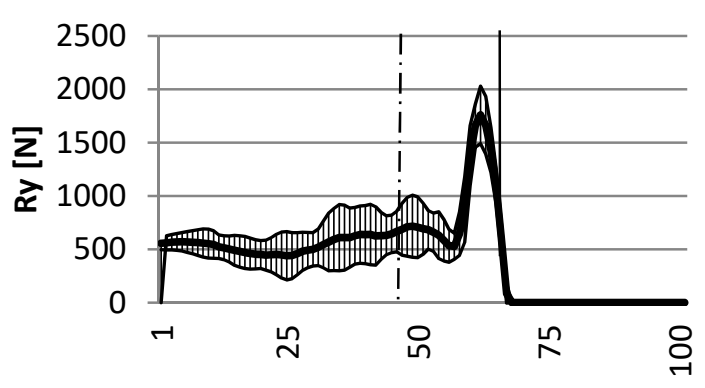

Time [\%] b. Handspring

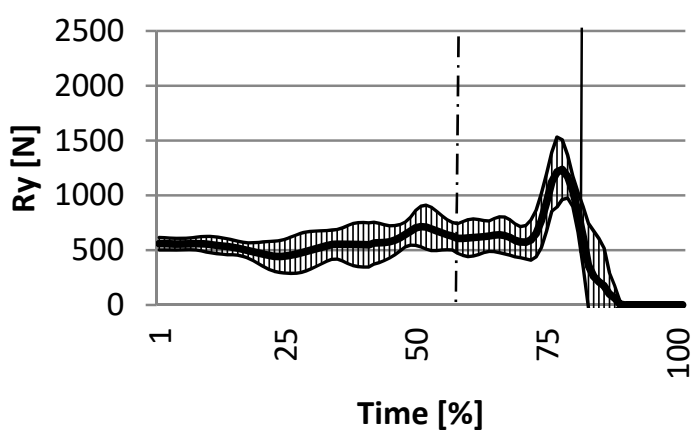

Figure 6. External-kinetic structure of the movement - the averaged and temporarily normalized vertical ground reaction force $-R_{y}[N]$ during the standing tucked backward somersault (a) and the standing backward handspring (b). Further explanations as in Figure 1. 
a. Tucked somersault

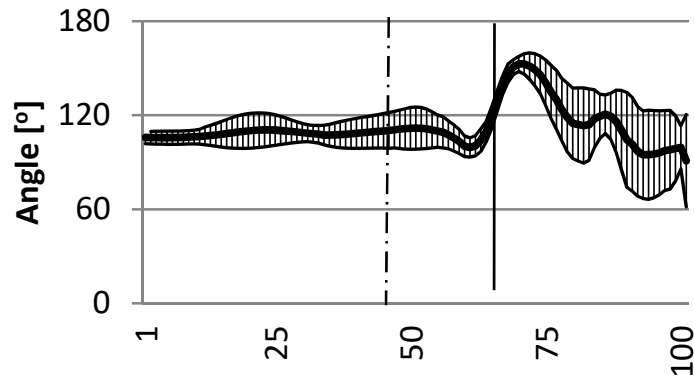

Time [\%] b. Countermovement jump

Ankle joint

\section{b. Countermovement jump}

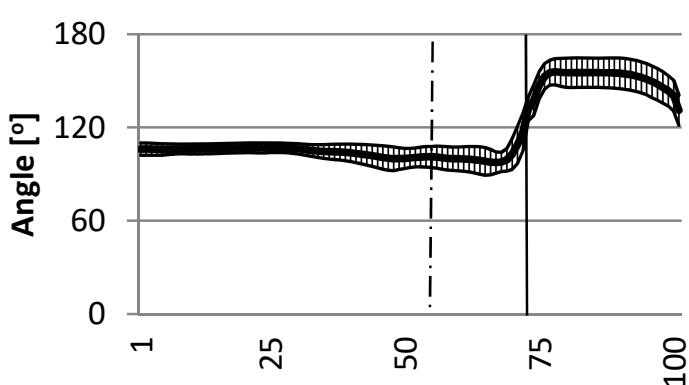

Time[\%]

Knee joint

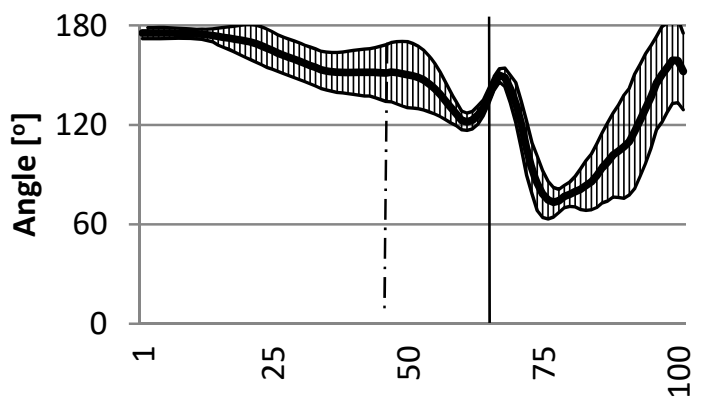

Time [\%]

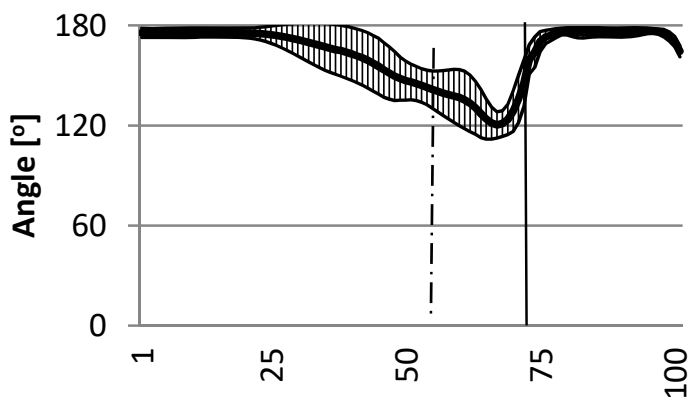

Time [\%]

Hip joint
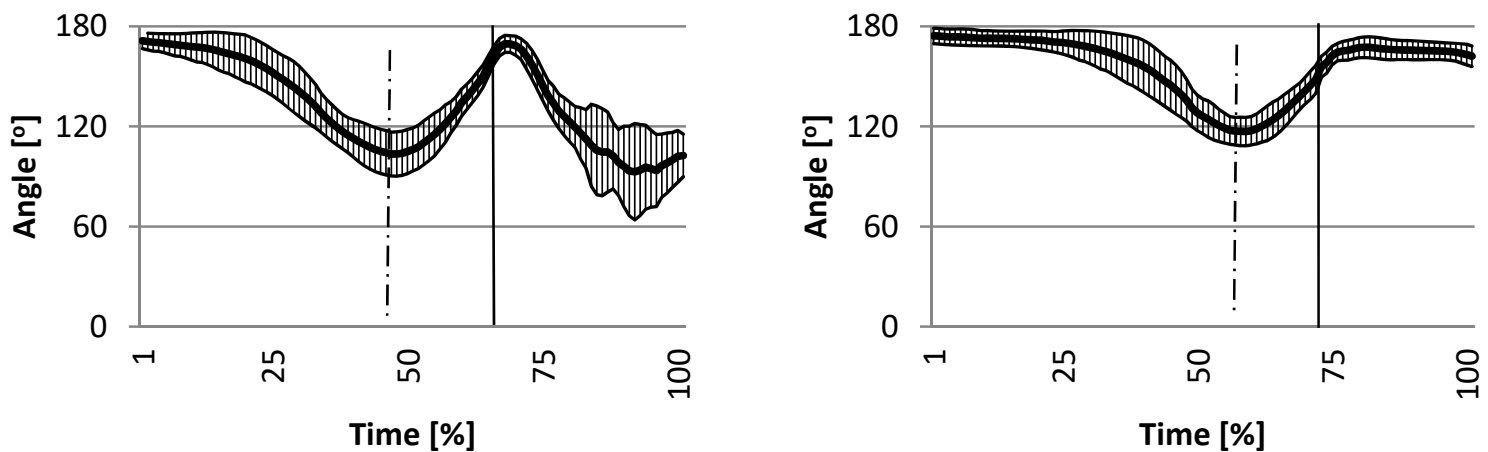

Time [\%]

Shoulder joint

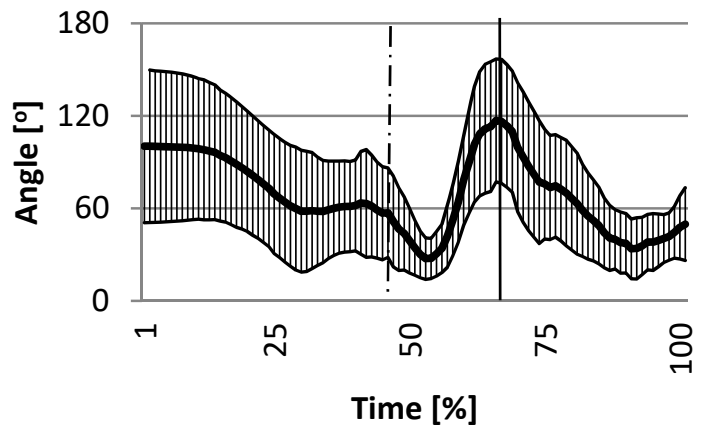

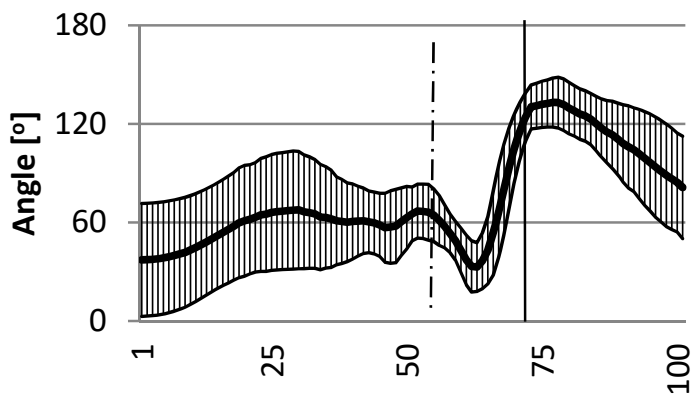

Time [\%]

Figure 7. External-kinematic structure of the movement - the averaged and temporarily normalized angle-time curves $\left[^{\circ}\right]$ for the relative angles of upper and lower leg joints during the standing tucked backward somersault (a) and the countermovement jump (b). Further explanations as in Figure 1. 


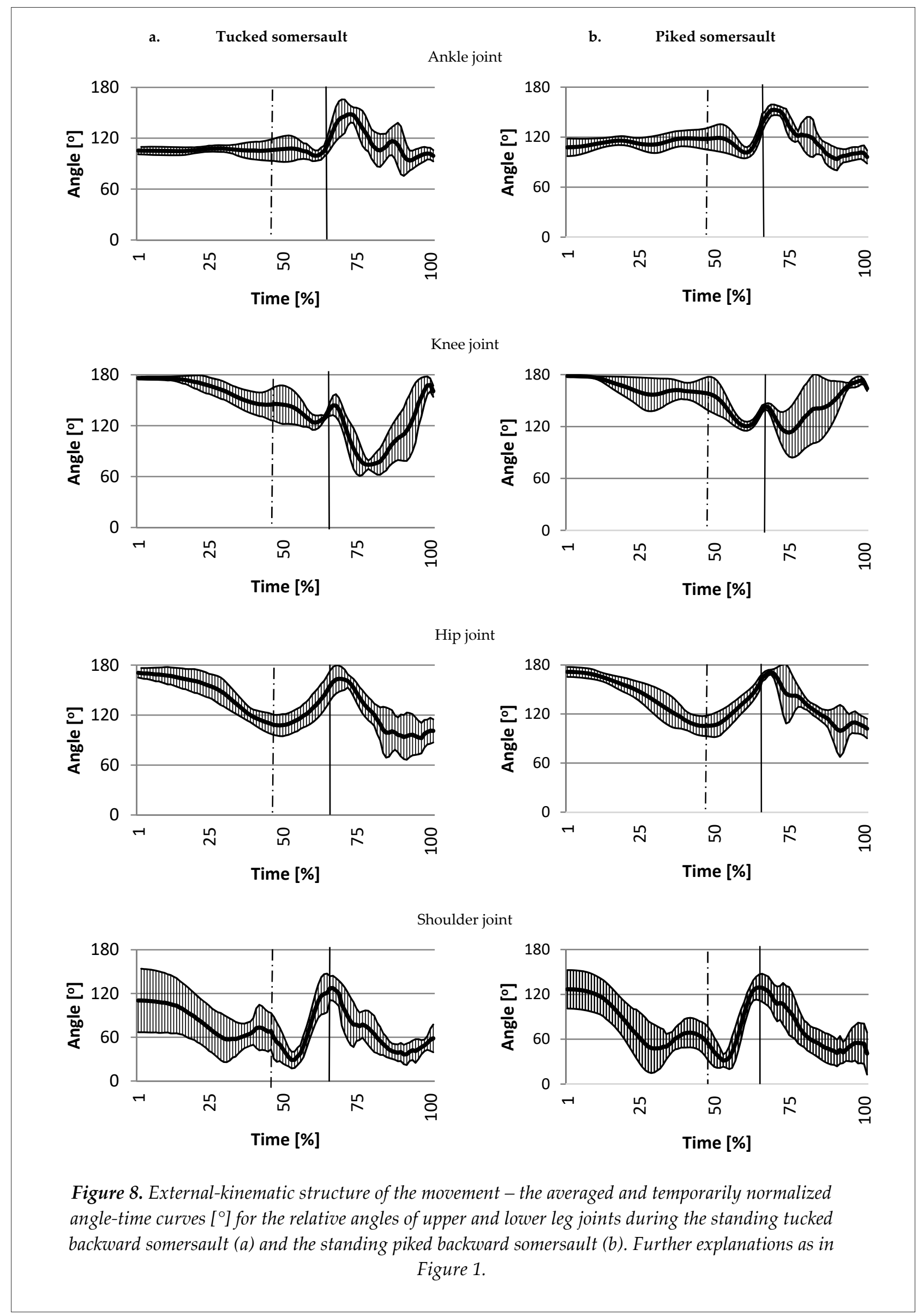




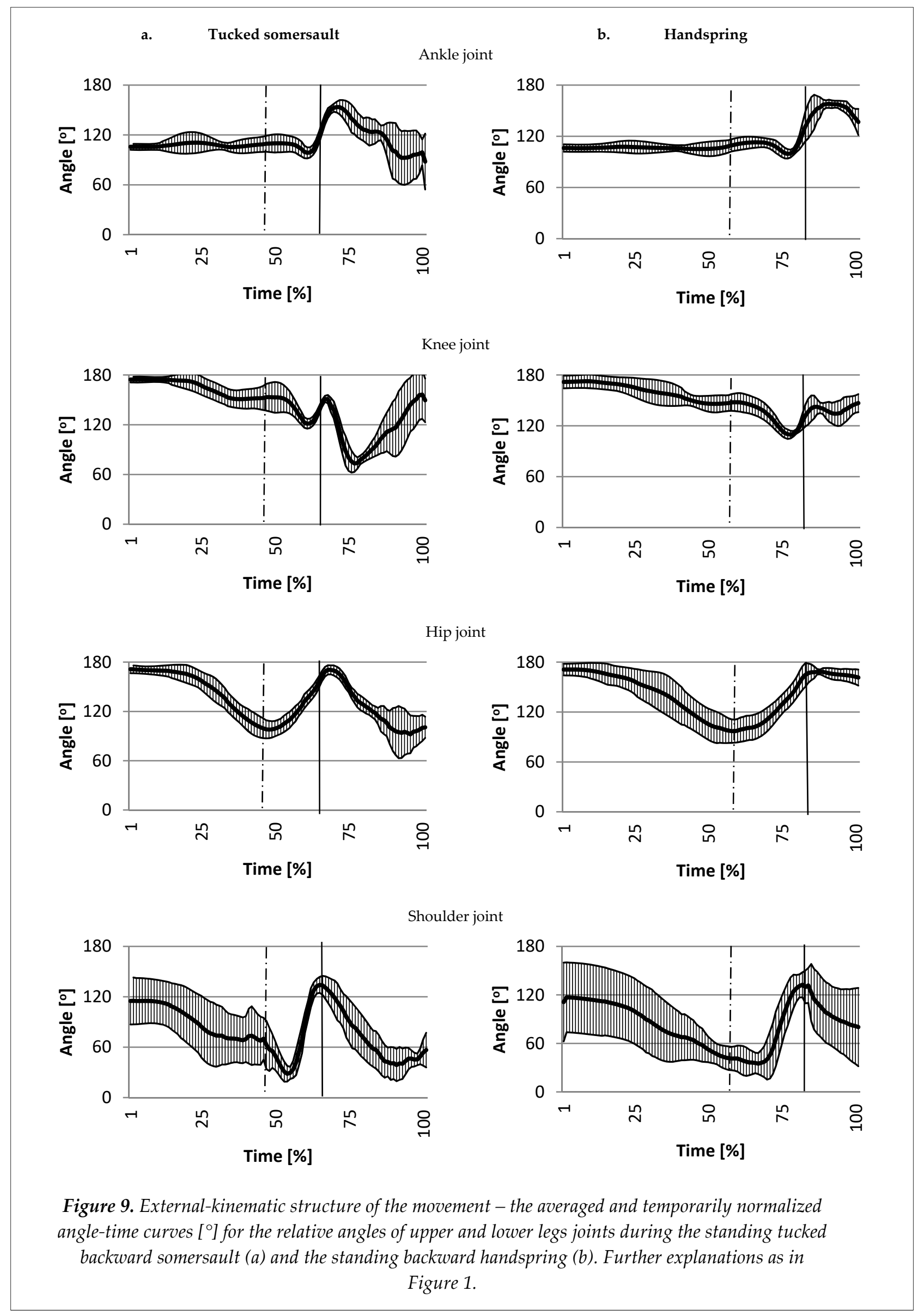


As expected, there were no statistically significant differences between the tucked and piked backward somersault in muscle activation levels of all eight muscles during the three successive phases.

There were statistically significant differences $(p<0.05)$ between the TS and HS in mean muscle activation levels of almost all muscles in the flight phase. In one muscle (medial gastrocnemius) a statistical tendency $(p=0.0687)$ was found. The high muscle activation levels of almost all muscles are probably associated with the stabilization of joints during the flight phase in the HS.

Changes in the myoelectrical activity of the prime movers may be associated with the take-off angle in particular jumps (Table 4). Decreasing the take-off angle in the TS, PS and HS, respectively, in comparison to the CMJ $(\mathrm{p}<0.05)$ corresponded to greater activity of the biceps femoris and erector spinae, and less activity of the rectus femoris. Although the differences between average muscle activation levels of these prime movers were usually small and the standard deviations large, with respect to the impulse values from ground reaction forces (Table 4 and Results) one should suppose that these muscles are fundamental in shaping the take-off mechanism.

It is important to note that to jump vertically, there should be no horizontal component of the GRF. In fact, in order to induce the backward rotation of the body, the vertical push's centre of pressure (COP) should be in front of the body's COM (De Jaeger et al., 2004). However, if the direction of the force is not towards the COM this could lead to some 'wasted effort' and therefore, insufficient height may be reached. Is it even possible to exert a vertical push which is not directed towards the COM without inducing a horizontal force? To answer this question, the values of horizontal and vertical components of the GRF would have to be evaluated.

A comparison of the acrobatic jumps with rotation showed that in the take-off phase of the TS a significantly higher vertical impulse was created than in the PS and especially in the HS. Obviously, in the countermovement jump the value of the vertical impulse was the greatest. Leboeuf et al. (2003) mentioned that if a backward somersault was performed correctly, the force impulse would be around $200 \mathrm{~N} \cdot \mathrm{s}$. Their values were much higher than those obtained in the current study. Our female gymnasts' impulse ranged between 133.6 $\pm 28.4 \mathrm{~N} \cdot \mathrm{s}$ and $128.1 \pm 25.4 \mathrm{~N} \cdot \mathrm{s}$ for the TS and PS, respectively (a comparison was performed for the same group of gymnasts). This difference may also be related to the fact that our gymnasts performed standing backward somersaults whereas in the Leboeuf's et al. (2003) study, they performed it after a descending jump.

The opposite was found with the horizontal impulse. The smallest magnitudes of the horizontal force impulse were found in the countermovement jump and in the tucked somersault, followed by the piked somersault and handspring, respectively. As expected, the horizontal displacement was the largest during the HS, caused by a take-off of the COM and allowing rotation, as described by Medved et al. (1995) and Munkasy et al. (1996).

The magnitude of the horizontal displacement, the flight height and the velocity of the COM which was reached at the end of the takeoff phase result from the force impulses. Of course, the obtained values of these variables are arranged as in the case of the vertical and horizontal impulses.

In our study, the values of the COM vertical velocity are about half that of Hraski's (2002) results. In Hraski's research, however, the backward somersault was performed after a typical acrobatic sequence: a run up, a round-off, a backward handspring, and the subject was a highly ranked, world class gymnast.

According to Lukjan and Parlak (2005), the smaller magnitude of vertical velocity, and thus, the flight height in somersaults, compared with the countermovement jump, are affected by:

- shortening the displacement of the COM of the body during the take-off phase, resulting from a shallower squat,

- extension (straightening) of the hip preceding the straightening of the knee joints,

- incomplete knee joint extension at the end of the take-off phase,

- shortening the time of the take-off phase.

Commencing the take-off phase by extending the hip joints before the knee joints and incomplete knee joint extensions at the beginning of the flight phase of the somersault was also confirmed in our study (Figure7). There were some differences, however, in the duration of 
subsequent phases, which may have resulted from the slightly different method of determining the beginning and the end of the take-off phase. In our study the beginning of the take-off phase was accepted as the moment of the return of the movement in the hip joints, after the countermovement phase. The end of this phase was accepted as the moment that the feet lost contact with the ground. In a study by Lukjan and Parlak (2005) the probable beginning of the take-off phase was at the lowest momentary location (position) of the COM at the end of the countermovement phase. The temporal ratio of the successive phases (the rhythm of the movement; Schnabel, 1998), as could be anticipated, was different for each of the jumps (Table 5).This was the most evident in the backward handspring. In the HS, the take-off phase was clearly longer, while the flight phase significantly shorter (almost two times shorter) than in the somersault and the countermovement jump.

The difference between the flight height in the standing countermovement jump and the standing somersault may be an indicator of the efficiency of the mechanism giving the angular momentum (Lukjan and Parlak, 2005). It can be assumed that the smaller this difference, the better the take-off to perform a standing somersault. In this way, the mechanism giving the angular momentum, which results from the similar nature of the take-off in both forms of jumping, is understood.

Besides the vertical velocity of the COM the angular momentum is another critical feature of the movement, and tends to be reversed. The great magnitude of the vertical velocity in the tucked backward somersault corresponds to the small angular momentum, while the small magnitude of the vertical velocity in the piked backward somersault corresponds to the high angular momentum. The magnitude of the angular momentum attributed to the COM at the take-off phase was determined during the flight phase immediately after the feet lost contact with the ground.

The angular momentum achieved by gymnasts in our research for the standing tucked somersault was $53.1 \pm 8.2 \mathrm{kgm} 2 / \mathrm{s}$, which was about $5.1 \mathrm{kgm} 2 / \mathrm{s}$ more than in the study by Lukjan and Parlak (2005). In the standing piked somersault, gymnasts achieved an angular momentum of about $19.9 \mathrm{kgm} 2 / \mathrm{s}$ greater than the athlete in the Lukjan and Parlak's study (2005) in the tucked somersault.

In the study by Hwang et al. (1990), the magnitudes of the angular momentum were more than twice our results. However, in their study, seven top-class athletes performed the double somersault after a running start. The results of our study are consistent with the Hraski's thesis (2002) which states that greater angular momentum during the flight phase corresponds to greater horizontal velocity and smaller vertical velocity during the take-off phase.

\section{Conclusions}

As expected, the take-off that passed through the COM allowed for a better amplitude of movement than the take-offs thrown off the centre forward or backward. The CMJ showed the highest level of vertical impulse, velocity, and displacement followed by the TS, PS, and HS. This implies that for better performance of acrobatic jumps, it is necessary that the force passes close to the COM. This explains why in the take-off phase of acrobatic jumps with rotation (TS, PS and HS) the average muscle activation levels of the biarticular biceps femoris were significantly higher (a prime mover) and of the biarticular rectus femoris significantly lower (a stabilizer) than in the countermovement jump (CMJ). Thus, the hypothesis accepted in the introduction was confirmed. In practice, we recommend that coaches carefully monitor the position of a gymnast's shoulders. Leaning backwards during the take-off while performing a standing backward acrobatic jump with rotation should be avoided.

Finally, although many variables influence sports success, including mental and physiological factors, biomechanical considerations reflected in correct or incorrect technique are crucial. This is especially true in such sports as gymnastics or figure skating. Despite the extensive use of acrobatic jumps in many forms of training regimes, there is still a paucity of published research directed toward the mechanical understanding of these movements. Gymnasts in particular, must master the following technical skills since they are included in most acrobatic jumps/skills: the ability to gain height, the ability to rotate, increasing or decreasing rotation by altering body configuration.

To better understand the cause-and-effect relationships between biomechanical factors, a 
computer simulation is often used (Yeadon et al., 1990). This is done through systematic manipulations of the key performance factors (critical features e.g. the vertical velocity of the $\mathrm{COM}$, the take-off angle, and the angular momentum around the COM at the moment of the take-off). The quality of the simulation results depends on the accuracy of the input data and the complexity of the model used. This first factor depends, inter alia, on the class of the measuring devices. In our study, we used modern comprehensive research methodology consisting of eight pairs of surface electrodes, six infrared cameras and a force plate, all of which were synchronized with each other (multi-modular measuring system SMART-E). This system allowed the internal and external structure of acrobatic jumps to be explored. The often subtle differences between the mechanical variables of motion can also be recorded and a technique assessment can be conducted. Sometimes just small differences that cannot be initially noticed may strongly influence performance of certain gymnastic skills.

\section{Acknowledgements}

This study was made possible by the financial support of the Ministry of Science and Higher Education of Poland and the Jerzy Kukuczka Academy of Physical Education in Katowice. The authors would like to thank Dr. Grzegorz Sobota for his work with data collection and compiling.

\section{References}

Bartlett R. Introduction to sports biomechanics: Analysing Human Movement Patterns (second edn.). London, Routledge; 2009

Bobbert MF, van Ingen Schenau GJ. Coordination in vertical jumping. J Biomech, 1988; 21: 249-262

Bruggemann G-P. Biomechanics of gymnastic techniques. Sport Sci Rev,1994; 3: 79-120

De Jaeger D, Vandervetde L, Willems PA. Kinematics of standing back-somersaults. XXIX Congress of the Biomecanice Society, Creteil; 2004

Droszez A, Sanno M, Goldmann J-P, Albracht K, Bruggemann G-P, Braunstein B. Differences between take-of behavior during vertical jumps and two artistic elements. XXXIV International Conference of Biomechanics in Sport, Tsukuba, Japan2016; 577-580

Ebig M, Lephart SM, Burdett RG, Miller MC, Pincivero DM. The effect of sudden inversion stress on EMG activity of the peroneal and tibialis anterior muscles in the chronically unstable ankle. J Orthop Sports Phys Ther, 1997; 26: 73-77

Geiblinger H, Morrison WE, McLaughlin PA. Take-off characteristics of double somersaults on the floor. In: Bauer T. (Ed.), Proceedings of the XIII International Symposium on Biomechanics in Sports, Thunder Bay, Lakehead University, 1995;128-131

George GS. Championship Gymnastics: Biomechanical Techniques for Shaping Winners. Carlsbad, CA: Designs for Wellness Press; 2014

Gola A, Krol H. Biomechanical analysis of flat bench pressing (case study). In: Urbanik C, Mastalerz A, Iwanska D. (Eds.) Selected problems of biomechanics of sport and rehabilitation vol II, Warsaw, Jozef Pilsudski University of Physical Education, 2014; 32-42

Hedbavny M, Kalichova M. Analysis of take-off phase of somersaults with twisting along the longitudinal body axis. Word Acadf Sci, Engineer Technol, 2011; 59: 590-593

Hraski Z. Correlation between selected kinematic parameters and angular momentum in backward somersaults. In: GianikellisKE. (Ed.), Proceedings of the XX International Symposium on Biomechanics in Sports, Caceres, University of Extramadura, 2002; 167-170

Hwang I, Seo G, Liu ZG. Take-off mechanics of the double backward somersault. Int J Sport Biomech,1990; 6:177-186 
Jacobs R, Bobbert MF, van Ingen Schenau G. Mechanical output from individual muscles during explosive leg extensions: the role of biarticular muscles. J Biomech, 1996; 29:513-523

Knoll K, Krug J. Floor: Men and Women. In: Bruggemann G-P, Krug J. (Eds.), The word championships in artistic gymnastics: Scientific report. Cologne, German Sport University, 1989

Konrad P. The ABC of EMG: A practical introduction to kinesiological electromyography. Version 1.4 March 2006, Noraxon Inc. USA; 2006

Kristofic J. Physical aspects of sport technique. Praha, Karolinum; 1996

Krol H, Golas A. Effect of barbell weight on the structure of the flat bench press. J Strength Cond Res, 2017; 31(5): 1321-1337

Krol H, Klyszcz-Morciniec M. Kinetic and kinematic characteristics as the basic for evaluating take-off in backward acrobatic jumps. Pol J Sport Turism, 2017; 24:139-144

Krol H, Golas A, Sobota G. Complex analysis of movement in evaluation of flat bench press performance. Acta Bioeng Biomech, 2010;12: 94-98

Król H, Klyszcz-Morciniec M, Sobota G. Takeoff mechanics of the acrobatic tumbling exercises (case study). In: Urbanik C, Mastalerz A, Iwańska D. (Eds.) Selected problems of biomechanics of sport and rehabilitation vol II, Warsaw, Jozef Pilsudski University of Physical Education, 2014; 62-75

Krol H, Klyszcz-Morciniec M, Sobota G, Nowak K. The complex analysis of movement in the evaluation of the backward somersault performance. Phys Act Rev, 2016; 4: 28-39

Leboeuf F, Lacouture P, Bessonet G. Dynamic analysis of an impulse movement in gymnastics. Proceedings of the IX Congress of ACAPS, Valence; 2003

Lukin MS. A method of analysis of athletic movements which are perfected without resistance positions. Theory Pract Phys Cult, 1964;10:26-28

Lukin MS. Standing backward somersault. Theory Pract Phys Cult,1966;12: 55-57

Lukjan M, Parlak J. Comparison of the take-off structure for counter movement jump and somersault (a case stydy). J Kinesiol Exerc Sci, 2005; 32: 51-58

McKinley P, Pedotti A. Motor strategies in landing from a jump: the role of skill in task execution. Experim Brain Res, 1992; 90: 427-440

McNeal JR, Sands WA, Shultz BB. Muscle activation characteristics of tumbling take-offs. Sports Biomech, 2007; 6: $375-390$

Medved V, Tonkovic S, Cifrec M. Simple neuro-mechanical measure of the locomotors skill: an example of backward somersault. Med Prog Technol, 1995;21: 77-84

Mkaouer B, Jemni M, Amara S, Chaabene H, Padulo J, Tabka Z. Effect of three technical arms swings on the elevation of the center of mass during a standing back somersault. J Hum Kinet, 2014; 40: 37-48

Mkaouer B, Jemni M, Amara S, Chaaben H, Tabka Z. Kinematic and kinetic analysis of counter movement jump versus two different types of standing back somersault. Sci Gym J, 2012; 4: 61-71

Munkasy BA, McNitt-Gray JL, Michele D, Welch MD. Kinematics prior to contact in landings preceded by rotation. In: Proceedings of the XX Annual Meeting of the American Society of Biomechanics,Atlanta; 1996

Okubo Y. Muscle activity during back tuck somersault. In: Tucker K, Butler B, Hodes P. (Eds.), Proceedings of the XIX Congress of the International Society of Electrophysiology and Kinesiology, Brisbane, University of Brisbane; 2012

Pandy MG, Zajac FE, Sim E, Levine WS. An optimal control model for maximum-height human jumping. $J$ Biomech, 1990; 23: 1185-1198

Pereira R, Machado M, Miragaya dos Santos M, Pereira LN, Sampaio-Jorge F. Muscle activation sequence compromises vertical jump performance. Serb J Sports Sci, 2008; 2: 85-90

Schnabel G. General characteristics of the movementas an expression of the movement coordination. In: Meinel K, Schnabel G. (Eds.), Teaching of the movement: Sports movements, Berlin, Sport Verlag, 1998; 74145 
van Soest AJ, Bobbert MF. The contribution of muscle proprieties in the control of explosive movements. Biol Cyb, 1993; 69:195-204

van Zandwijk JPV, Bobbert MF, Munneke M. Control of maximal and submaximal vertical jumps. Med Sci Sport Exer, 2000; 32: 477-485

Wikstrom EA, Tillman MD, Schenker S, Borsa PA. Failed jump landing trials: deficits in neuromuscular control. Sca J Med Sci Sports, 2008; 18: 55-61

Yeadon MR, Atha J, Hales FD. The simulation of aerial movement - IV: A computer simulation model. J Biomech, 1990; 23: 85-89

Zatsiorsky VM, Seluyanov VN. The biomechanics of human musculoskeletal. In: Morecki A. (Ed), Proceedings of the VII International Congress of Biomechanics, Baltimore, University Park Press;1981

\section{Corresponding author:}

\section{Bogdan Bacik,}

Institute of Sport Sciences,

The Jerzy Kukuczka Academy of Physical Education,

Katowice, Poland

E-mail: b.bacik@awf.katowice.pl 